\section{Multifractal analysis of selected rare-earth elements}

To cite this article: A Cummings et al 2001 J. Phys. B: At. Mol. Opt. Phys. 342547

View the article online for updates and enhancements.

\section{Related content}

- Signatures of quantum chaos in rare earth
elements:Il
A Cummings, G O'Sullivan and D M
Heffernan
- Uniform partitions and a dimensions
$\frac{\text { Spectrum for lacunarmeasures }}{\text { R Santoro, N M Maraldi, S Campagna et }}$
al.
- Multifractal structure in two dimensions in
proton - nucleus interactions at high
energy
Neeti Parashar

\section{Recent citations}

- Multifractal Analysis of Charged Particle
$\frac{\text { Multiplicity Distribution in the Framework of }}{\text { Renvi Entropy }}$
Swarnapratim Bhattacharyya et al
- Characterization of the structure and
$\frac{\text { eigenvalue spectra of the compound }}{\text { States of Sm IX }}$
D Kilbane et al
- Spectral fluctuation characterization of
$\frac{\text { random matrix ensembles through }}{\text { wavelets }}$

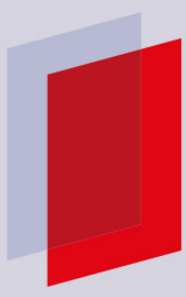

\section{IOP ebooks'}

Bringing you innovative digital publishing with leading voices

to create your essential collection of books in STEM research.

Start exploring the collection - download the first chapter of every title for free. 


\title{
Multifractal analysis of selected rare-earth elements
}

\author{
A Cummings ${ }^{1,2}$, G O'Sullivan ${ }^{1}$, W G Hanan ${ }^{2}$ and D M Heffernan ${ }^{2,3}$ \\ ${ }^{1}$ Physics Department, University College Dublin, Belfield, Dublin 4, Ireland \\ 2 Department of Mathematical Physics, National University of Ireland Maynooth, Maynooth, \\ County Kildare, Ireland \\ ${ }^{3}$ School of Theoretical Physics, Dublin Institute for Advanced Studies, Dublin 4, Ireland
}

Received 17 January 2001, in final form 2 May 2001

\begin{abstract}
The multifractal formalism is applied to the energy eigenvalues of $\mathrm{Ce}$ I, $\mathrm{Ce}$ II, $\mathrm{Nd}$ II, Sm I, Sm II, and Tb I. The Rényi dimensions $D_{q}$, mass exponents $\tau(q)$ and $f(\alpha)$ spectra are calculated and used to characterize the eigenvalue spectra. It is found that these elements show multi-scaling behaviour that can be accurately modelled by simple multifractal recursive Cantor sets. The effect of unfolding the spectra is also investigated.
\end{abstract}

\section{Introduction}

Ever since the work of Mandelbrot [1,2] the scaling behaviour of numerous systems, both mathematical and physical, has been investigated. Examples include Rayleigh-Bénard thermal convection [3], diffusion-limited aggregation [4], cluster-cluster aggregates [5], epithelial cell nuclei [6], heartbeat dynamics [7] and eigenvector components [8]. The results of these studies have all concluded that many of the systems not only have fractal properties but are, in fact, multifractals and so possess multi-scaling properties.

In this paper, the multifractal analysis of the energy levels of various lanthanide atoms and ions, namely Ce I, Ce II, Nd II, Sm I, Sm II and Tb I, is performed. Previous investigations [9-11] of atomic systems have used the box-counting method of [12]. However, the generalized correlation sum of [13] will be used here:

$C_{q}^{d}(R)=\lim _{\substack{R \rightarrow 0 \\ N \rightarrow \infty}}\left\{\frac{1}{N} \sum_{i=1}^{N}\left(\frac{1}{N} \sum_{j=1}^{N} \Theta\left(R-\left[\sum_{n=0}^{d-1}\left(x_{i+n}-x_{j+n}\right)^{2}\right]^{1 / 2}\right)\right)^{q-1}\right\}^{1 /(q-1)}$

where the Heaviside function $\Theta$ counts how many pairs of points $\left(x_{i}, x_{j}\right)$ are situated within a distance $R$ and $d$ is the embedding dimension.

In section 2 the Rényi dimensions $D_{q}$, mass exponents $\tau(q)$ and $f(\alpha)$ spectra are calculated for $\mathrm{Ce}_{\mathrm{I}}$ and $\mathrm{Ce}$ II, and similarly for $\mathrm{Nd}$ II, Sm I, Sm II and Tb I in section 4. For these elements, the closeness of the $4 \mathrm{f}, 6 \mathrm{~s}$ and $5 \mathrm{~d}$ binding energies gives rise to high level densities. In the case of $\mathrm{Ce}$, the similarity of the statistical properties of the electronic states and transitions between them, with those arising in compound nuclei have been detailed and attributed to chaotic behaviour $[14,15]$. 

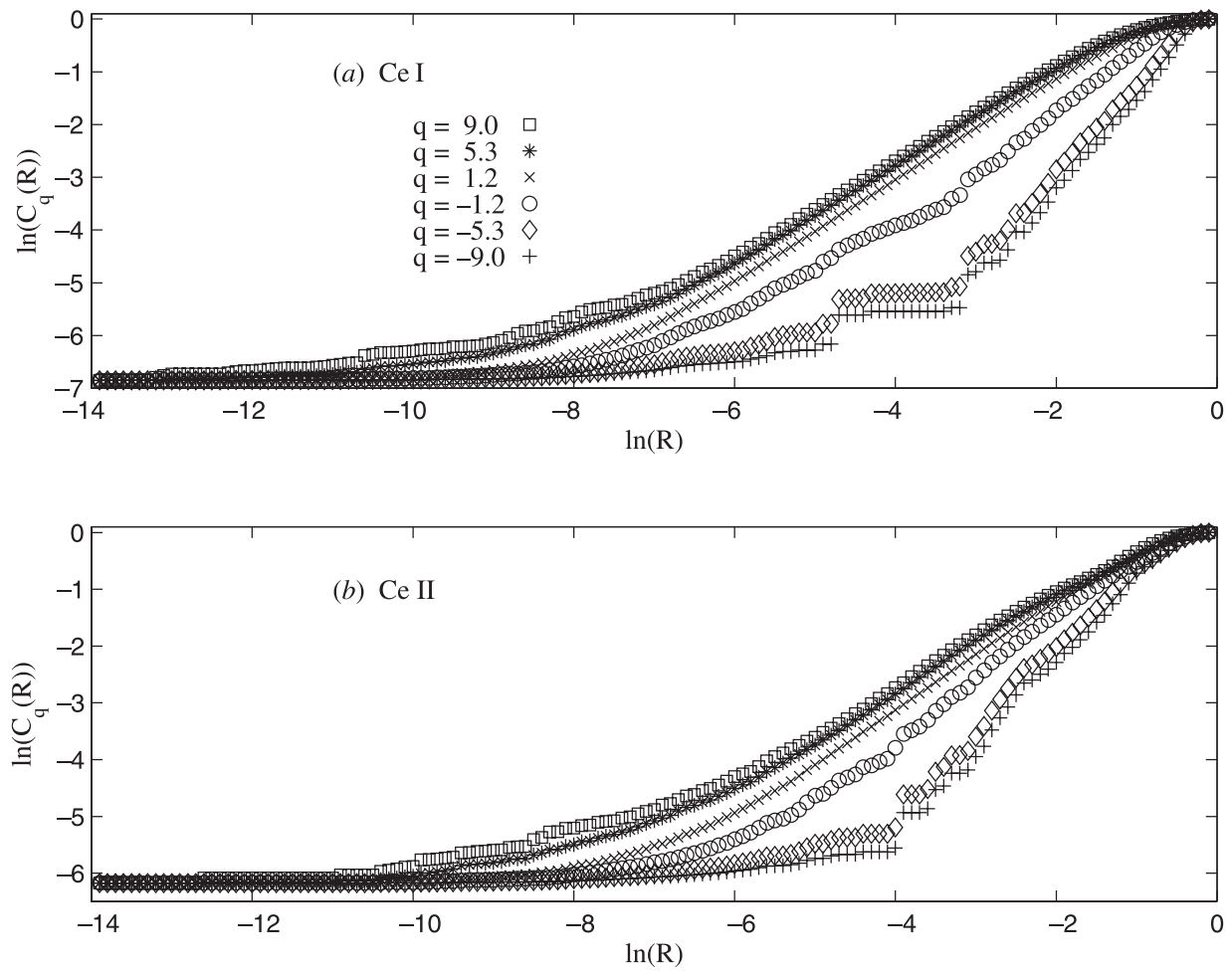

Figure 1. $\operatorname{Ln}\left(C_{q}(R)\right)$ versus $\ln (R)$ for $(a)$ Ce I and $(b)$ Ce II.

Table 1. Even and odd configurations of $\mathrm{Ce}_{\mathrm{I}}$ and $\mathrm{Ce}$ II.

\begin{tabular}{|c|c|}
\hline \multicolumn{2}{|l|}{$\mathrm{Ce}_{\mathrm{I}}$} \\
\hline Ground configuration & $1 s^{2} 2 s^{2} 2 p^{6} 3 s^{2} 3 p^{6} 3 d^{10} 4 s^{2} 4 p^{6} 4 d^{10} 5 s^{2} 5 p^{6} 4 f 5 d 6 s^{2}$ \\
\hline Odd configurations & $4 f 5 d 6 s^{2}, 4 f 5 d^{2} 6 s, 4 f 5 d^{3}, 4 f^{2} 6 s 6 p$ \\
\hline Even configurations & $4 \mathrm{f}^{2} 6 \mathrm{~s}^{2}, 4 \mathrm{f}^{2} 5 \mathrm{~d} 6 \mathrm{~s}, 4 \mathrm{f} 6 \mathrm{~s}^{2} 6 \mathrm{p}, 4 \mathrm{f} 5 \mathrm{~d} 6 \mathrm{~s} 6 \mathrm{p}, 4 \mathrm{f} 5 \mathrm{~d}^{2} 6 \mathrm{p}, 4 \mathrm{f}^{2} 5 \mathrm{~d}^{2}$ \\
\hline \multicolumn{2}{|l|}{$\mathrm{Ce}_{\text {II }}$} \\
\hline Ground configuration & $1 s^{2} 2 s^{2} 2 p^{6} 3 s^{2} 3 p^{6} 3 d^{10} 4 s^{2} 4 p^{6} 4 d^{10} 5 s^{2} 5 p^{6} 4 f 5 d^{2}$ \\
\hline Odd configurations & $4 f 5 d^{2}, 4 f 5 d 6 s, 4 f 6 s^{2}, 4 f^{2} 6 p, 4 f^{3}$ \\
\hline Even configurations & $4 f^{2} 6 s, 4 f^{2} 5 d, 4 f 5 d 6 p, 5 d^{3}, 4 f 6 s 6 p 5 d^{2} 6 s, 5 d 6 s^{2}$ \\
\hline
\end{tabular}

The $f(\alpha)$ spectra for Ce I and Ce II are simulated in section 3 using simple recursive Cantor sets with two length scales and two probability scales and in section 5 the effect of unfolding (rescaling) the eigenvalue spectrum of $\mathrm{Ce}_{\mathrm{I}}$ is investigated.

\section{Analysis of Ce I and Ce II energy eigenvalues}

Pawelzik and Schuster's generalized correlation sum method [13] was first applied to a sequence of energy levels from the neutral cerium atom and its ion, members of the lanthanide series of elements. The eigenvalues that were used ranged in energy from 0 to $3.843 \mathrm{eV}$ for $\mathrm{Ce}_{\mathrm{I}}(Z=58)$ and 0 to $6.455 \mathrm{eV}$ for $\mathrm{Ce}_{\text {II }}[16]$. Of course, these energies are measured relative to the ground state of the atom or ion. In each case both even- and odd-parity levels were 

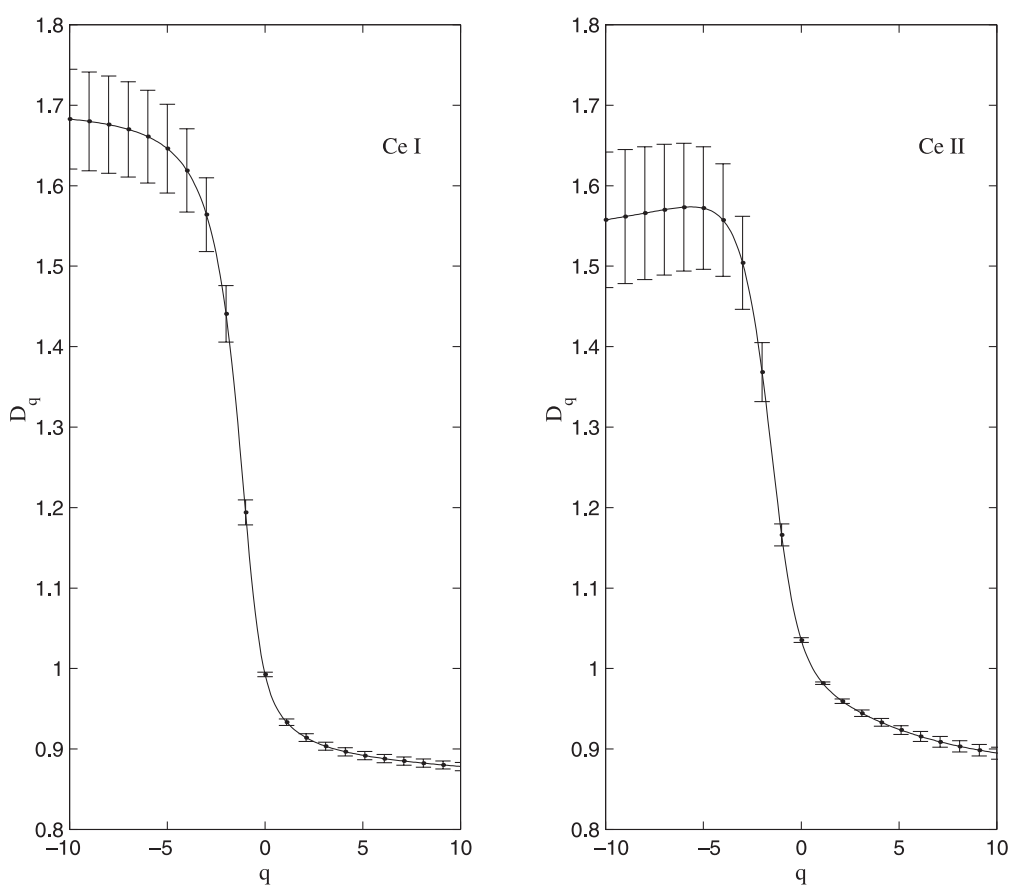

Figure 2. The generalized dimensions $D_{q}$ for Ce I and Ce II.

included as were all available $J$ values. A total of 943 and 480 energy states were used for Ce I and $\mathrm{Ce}$ II, respectively. The configurations that give rise to the energy eigenvalues are shown in table 1.

The correlation sum $C_{q}^{d}(R)$ for the following ranges of $q$ and $R$ :

$$
\begin{aligned}
& -20.0 \leqslant q \leqslant 20.0 \\
& -14.0 \leqslant \ln (R) \leqslant-0.1 \equiv 8.315 \mathrm{E}-7 \leqslant R \leqslant 0.9048
\end{aligned}
$$

was calculated. Note that the energy levels are re-scaled so that they lie between 0 and 1.0 when the generalized correlation sum is calculated. Plots of $\ln \left(C_{q}^{d}(R)\right)$ versus $\ln (R)$ for Ce I and Ce II with $d=1$ are shown in figures $1(a)$ and $(b)$, respectively. These plots clearly show that there is a region of scaling for both $\mathrm{Ce}_{\mathrm{I}}$ and $\mathrm{Ce}_{\mathrm{II}}$, but to quantify this scaling range more clearly the local slope of $\ln \left(C_{q}(R)\right)$ versus $\ln (R)$ was determined. The slope was calculated at every $\ln (R)$ for various $q$ and over a range of $\ln (R) \pm 0.4$.

Only for the following regions is the local slope found to be approximately constant:

$$
\begin{array}{ll}
-3.3 \leqslant \ln (R) \leqslant-1.7 \equiv 0.0369 \leqslant R \leqslant 0.1827 & \text { Cе I } \\
-3.8 \leqslant \ln (R) \leqslant-2.6 \equiv 0.0224 \leqslant R \leqslant 0.0743 & \text { Cе } \mathrm{II}
\end{array}
$$

and so are taken as the scaling regions (of course, the local slope fluctuations are highly dependent on the range of $\ln (R)$ that is used to calculate the slope). Therefore, the scaling ranges for $\mathrm{Ce}_{\mathrm{I}}$ and $\mathrm{Ce}_{\mathrm{II}}$ change in magnitude by factors of $0.1827 / 0.0369 \approx 4.95$ and $0.0743 / 0.0224 \approx 3.32$ for $\mathrm{Ce}_{\mathrm{I}}$ and $\mathrm{Ce}$ II, respectively. Thus $\mathrm{Ce}_{\mathrm{I}}$ scales over a much larger range than does Ce II.

Once these regions were found, the next task was to determine the generalized dimensions $D_{q}$. These are obtained from the slope of $\ln \left(C_{q}(R)\right)$ versus $\ln (R)$ over the scaling region. 

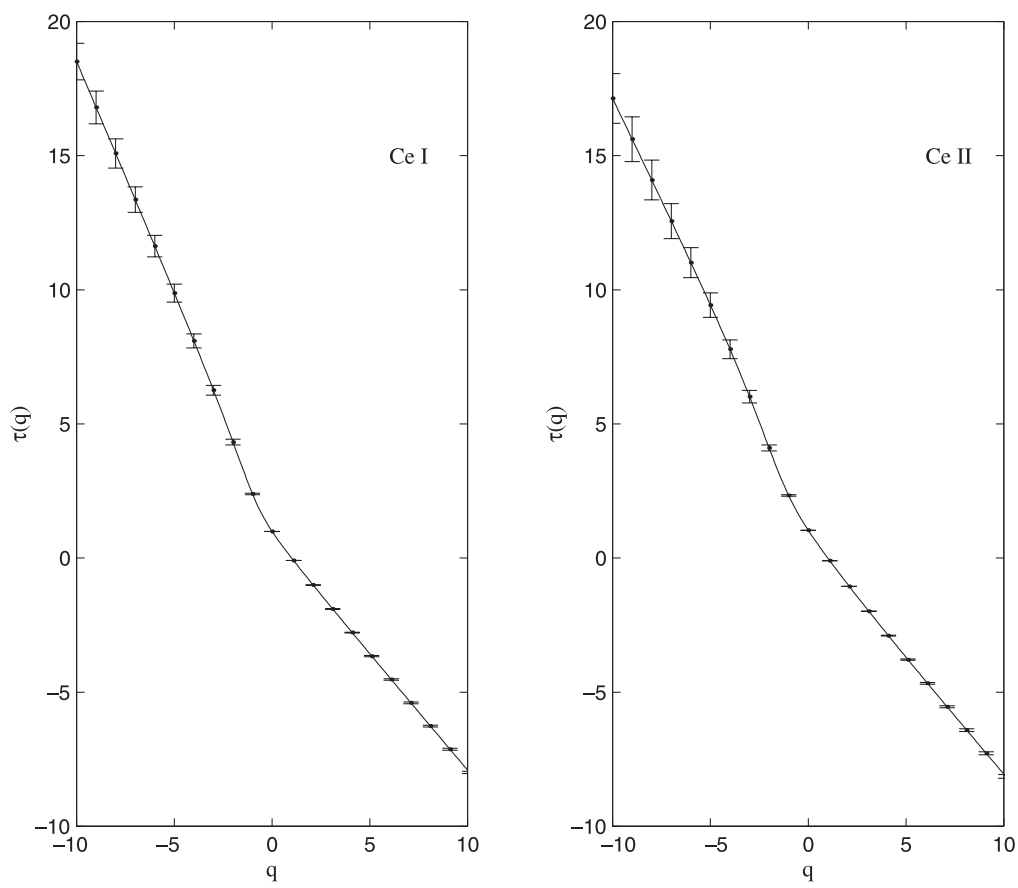

Figure 3. The sequence of mass exponents $\tau(q)$ for Ce I and Ce II.
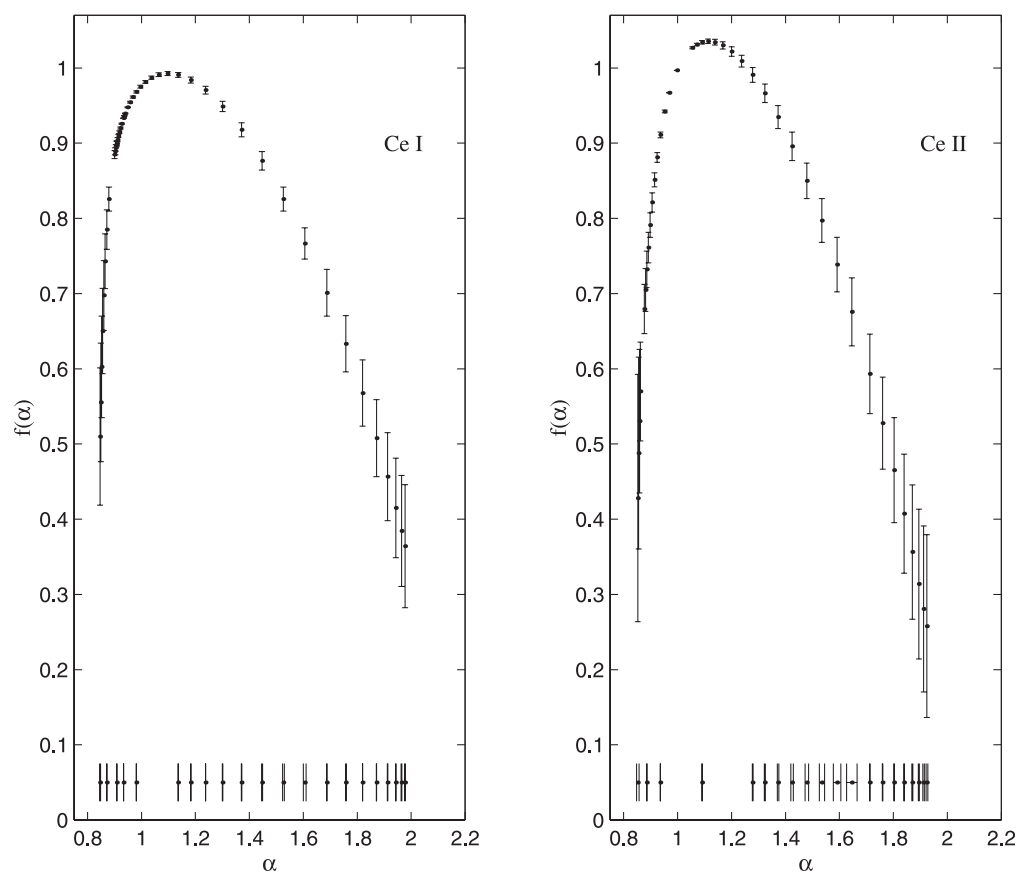

Figure 4. The $f(\alpha)$ spectra for $\mathrm{Ce}_{\mathrm{I}}$ and $\mathrm{Ce}$ II. 


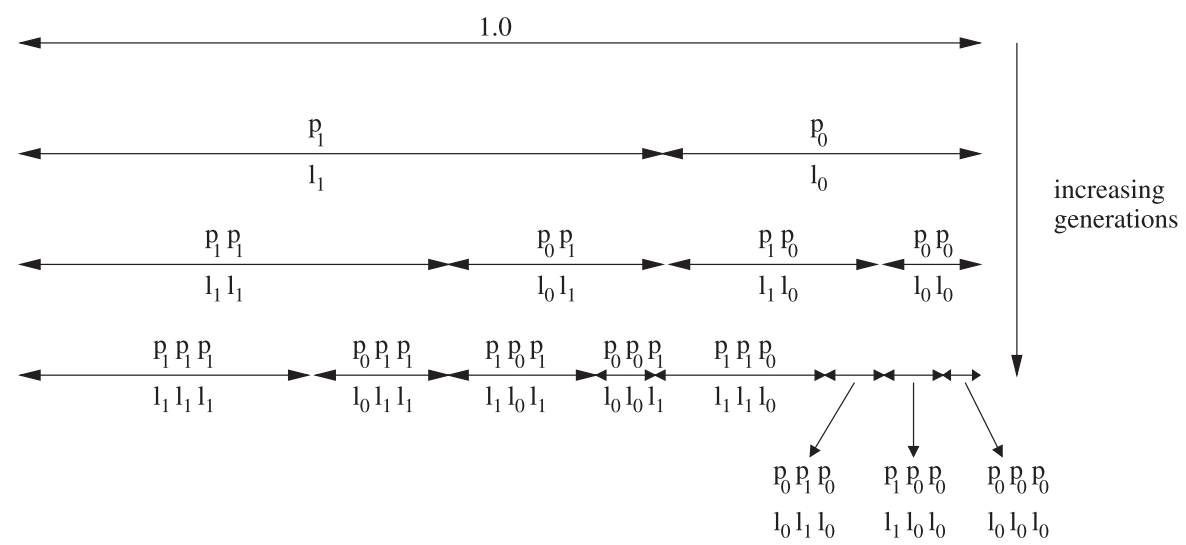

Figure 5. Schematic diagram of a binomial multiplicative process on the unit interval, with a continuous support for the measure.

$D_{q}$ with its corresponding error is shown in figure 2 for both Ce I and Ce II. $D_{q}$ should always be monotonically decreasing and in general, in the present case, $D_{q^{\prime}}>D_{q}$ for $q^{\prime}<q$, but there is a slight deviation from this when $q<-5.0$ for Ce II. However, from figure 2 it can be seen that the error limits are such as to allow for $D_{q^{\prime}}>D_{q}$ for all $q^{\prime}<q$.

It can be seen in figure 2 that the error in $D_{q}$ is greatest for large negative values of $q$. This is because negative $(q-1)$ probability moments used in the generalized correlation sum are more sensitive to small numbers of data points than are the positive $(q-1)$ probability moments.

Multiplying $D_{q}$ by $(1-q)$ gives the sequence of mass exponents $\tau(q)$ and these are shown in figure 3.

In order to determine the $f(\alpha)$ spectrum, a numerical Legendre transformation of $D_{q}$ was performed:

$$
\begin{aligned}
& \alpha(q)=\frac{\mathrm{d}}{\mathrm{d} q}\left[(q-1) D_{q}\right] \\
& f(\alpha(q))=q \frac{\mathrm{d}}{\mathrm{d} q}\left[(q-1) D_{q}\right]-(q-1) D_{q} .
\end{aligned}
$$

The resulting $f(\alpha)$ spectra for $\mathrm{Ce}_{\mathrm{I}}$ and $\mathrm{Ce}_{\mathrm{II}}$ are shown in figure 4, along with the corresponding error limits for both $f(\alpha)$ and $\alpha$.

Table 2 shows the range of $q$ values used in the Legendre transformation, the maximum and minimum values of the Lipschitz-Hölder exponents $\alpha$, as well as the Kolmogorov capacity $D_{0}$, information $D_{1}$ and correlation dimensions $D_{2}$ and the maximum value of $f(\alpha)$.

$D_{0}$ and the maximum value of $f(\alpha)$ for both $\mathrm{Ce}_{\mathrm{I}}$ and $\mathrm{Ce}$ II have values very close to 1.0 , which suggests that the support of the measure is one dimensional, i.e. a continuous 'line' of points/energy levels.

Since all of the calculations are sensitive to the number of data points and to any noise or gaps in the data, especially low density regions (negative $q$ ), the quality of the $f(\alpha)$ spectra obtained should only be viewed as tentative. Also, the scaling regions are quite limited and an interesting study of the dimensions of random distributions [17] shows that apparent fractal behaviour is observed for a range of scales typically spanning between one and two orders of magnitude - the typical range observed in experimental measurements of fractals. 

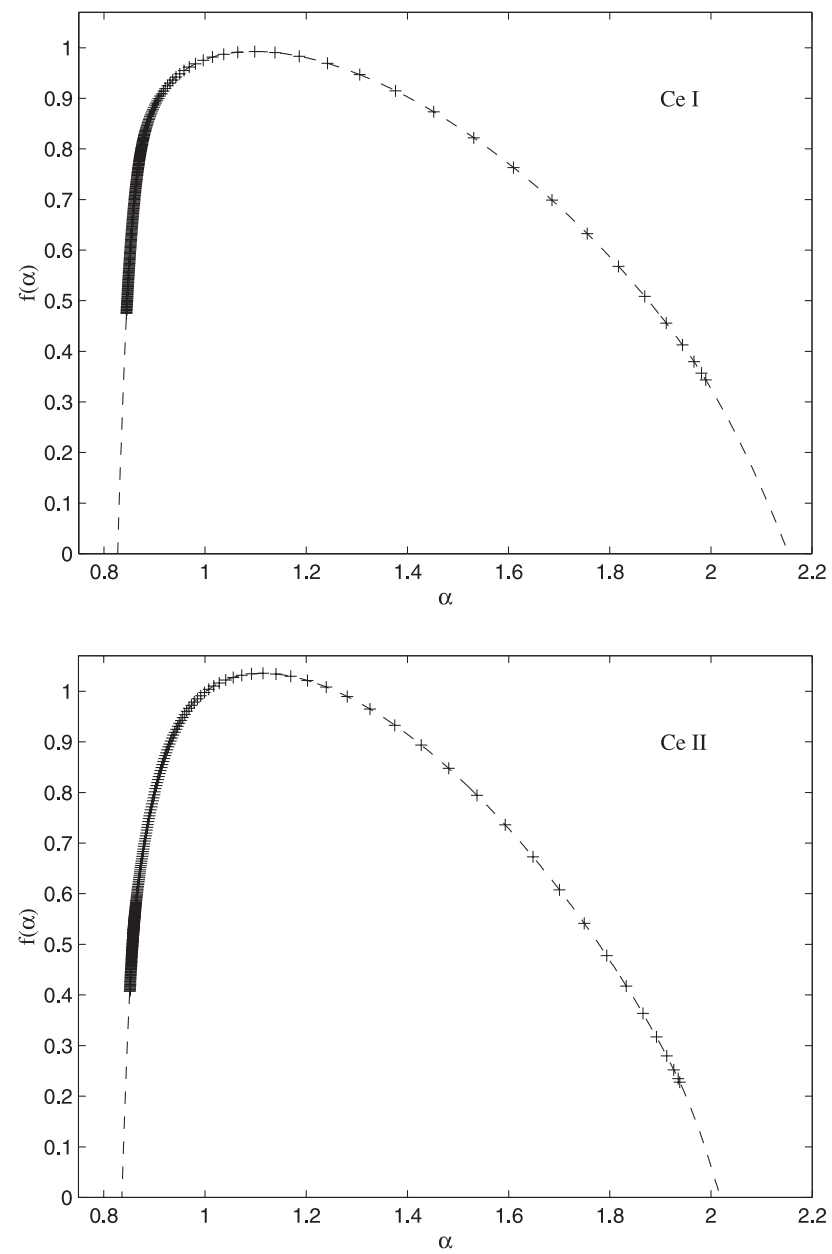

Figure 6. Interpolation of the $f(\alpha)$ spectra for Ce I and Ce II.

Table 2. Various $D_{q}$ and $f(\alpha)$ parameters for Ce I and Ce II.

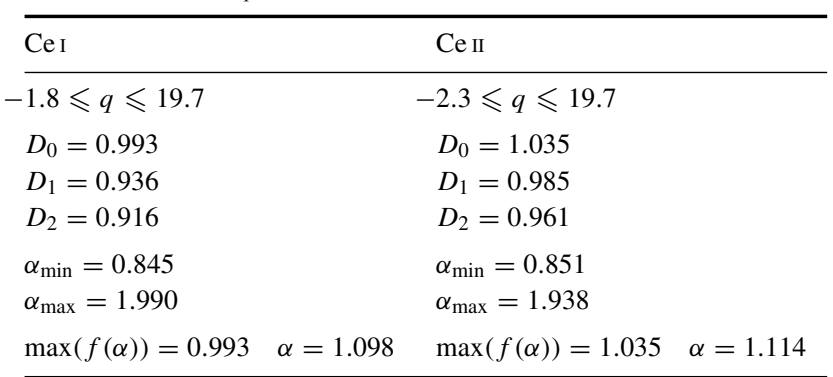

\section{3. $f(\alpha)$ simulation}

Having obtained multifractal spectra, it was decided to try to generate $f(\alpha)$ spectra that would have the same/similar form as the spectra obtained. In fact, there have been a number of 

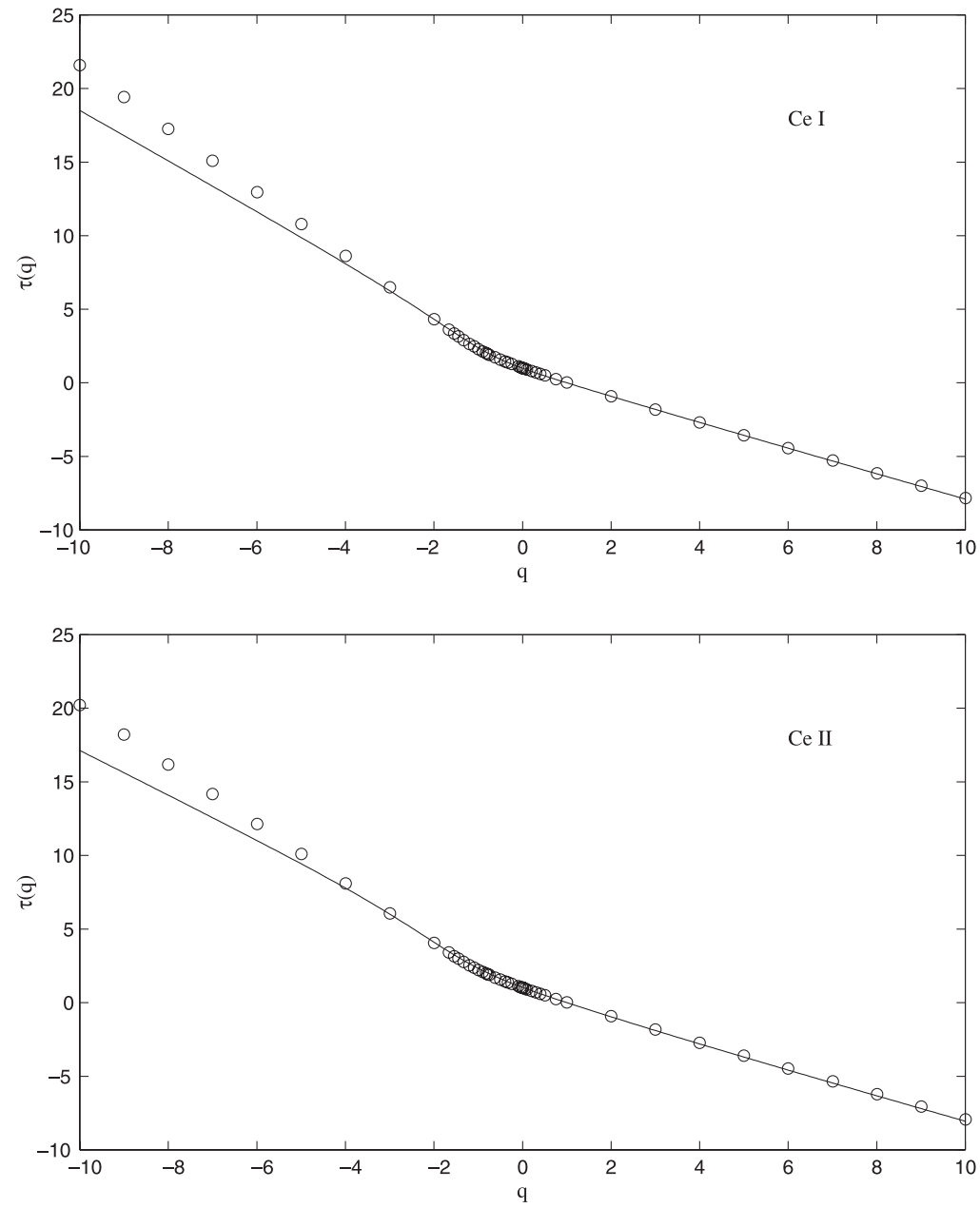

Figure 7. Comparison of 'experimental' (full curve) and 'numerical' (circles) $\tau(q)$.

attempts to discover the underlying scaling structure for a given $f(\alpha)$ spectrum [18,19]. Such attempts were found to be futile except in the case where the underlying fractal structure was built on recursive Cantor sets. Here it is shown that the scaling structure may be understood in terms of simple recursive Cantor sets which have two length scales and two probability scales. Why this should be the case is unclear, although for any configuration the exchange interaction will split the levels into groups depending on their multiplicity. Frequently, this results in the lowest multiplicity group, which contains relatively few levels, being split in energy from the higher multiplicity group(s) which contains the majority of levels of the configuration. Of course the question must be asked 'can a finite number of perturbations (e.g. $F^{k}, G^{k}, R^{k}$, spin-orbit, etc) account for multi-scaling behaviour over a large range of length scales?'. The multiplicative process with a continuous support (in this case the unit interval $[0,1]$ ) was assumed, since $D_{0} \approx 1.0$.

The initial underlying lengths and probabilities are shown schematically in figure 5 . The $f(\alpha)$ spectra were then interpolated, as shown in figure 6 , in order to find the 'theoretical' 

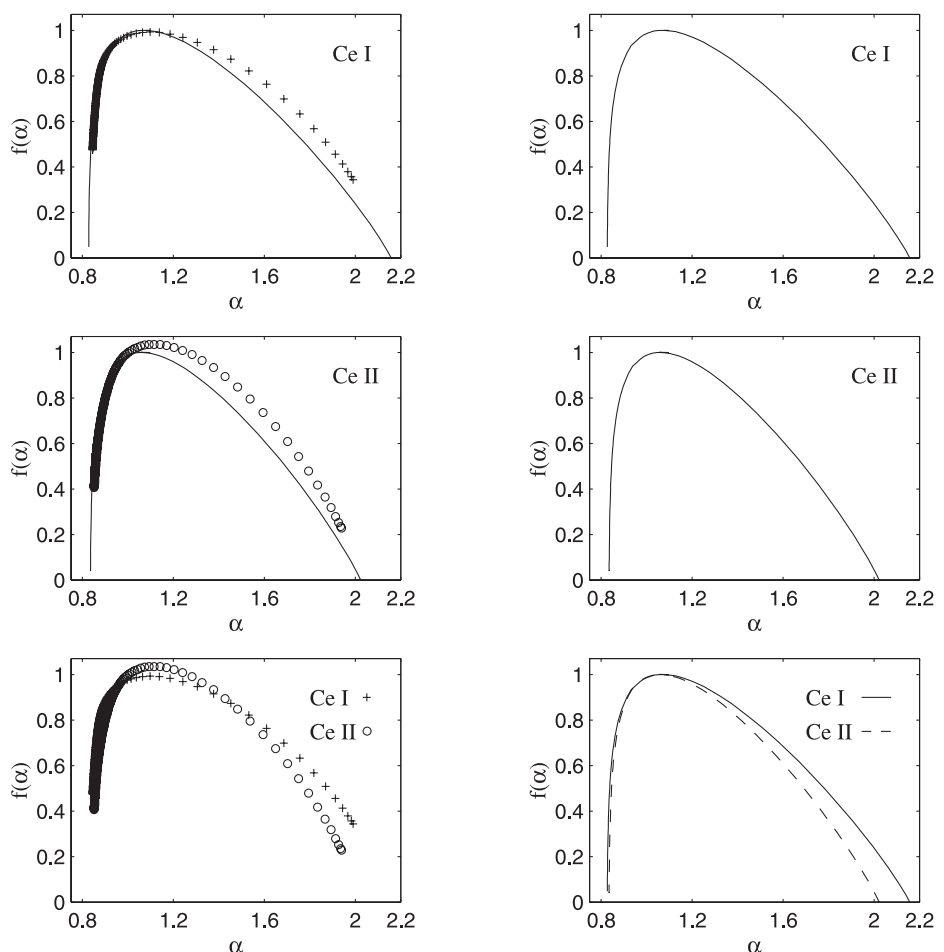

Figure 8. Comparison of the 'experimental' $(+, \circ)$ and 'numerical' $f(\alpha)$ spectra.

maximum and minimum $\alpha$ values. These values were substituted into the theoretical $\alpha_{\min }$ and $\alpha_{\max }$ of the two-scale fractal measure:

$$
\begin{gathered}
\alpha_{\text {min }}=\frac{\ln \left(p_{0}\right)}{\ln \left(l_{0}\right)} \\
\alpha_{\max }=\frac{\ln \left(p_{1}\right)}{\ln \left(l_{1}\right)}
\end{gathered}
$$

where a continuous one-dimensional support was assumed:

$$
l_{1}=1-l_{0}
$$

and of course

$$
p_{1}=1-p_{0}
$$

This is conservation of the probability measure.

Solving for $\mathrm{Ce}$ I and $\mathrm{Ce}$ II as follows:

$$
\begin{aligned}
\text { Ce I } & \alpha_{\text {min }}=0.828=\frac{\ln \left(p_{0}\right)}{\ln \left(l_{0}\right)} & \alpha_{\max }=2.154=\frac{\ln \left(p_{1}\right)}{\ln \left(l_{1}\right)} \\
\text { Ce II } & \alpha_{\min }=0.836=\frac{\ln \left(p_{0}\right)}{\ln \left(l_{0}\right)} & \alpha_{\max }=2.019=\frac{\ln \left(p_{1}\right)}{\ln \left(l_{1}\right)}
\end{aligned}
$$



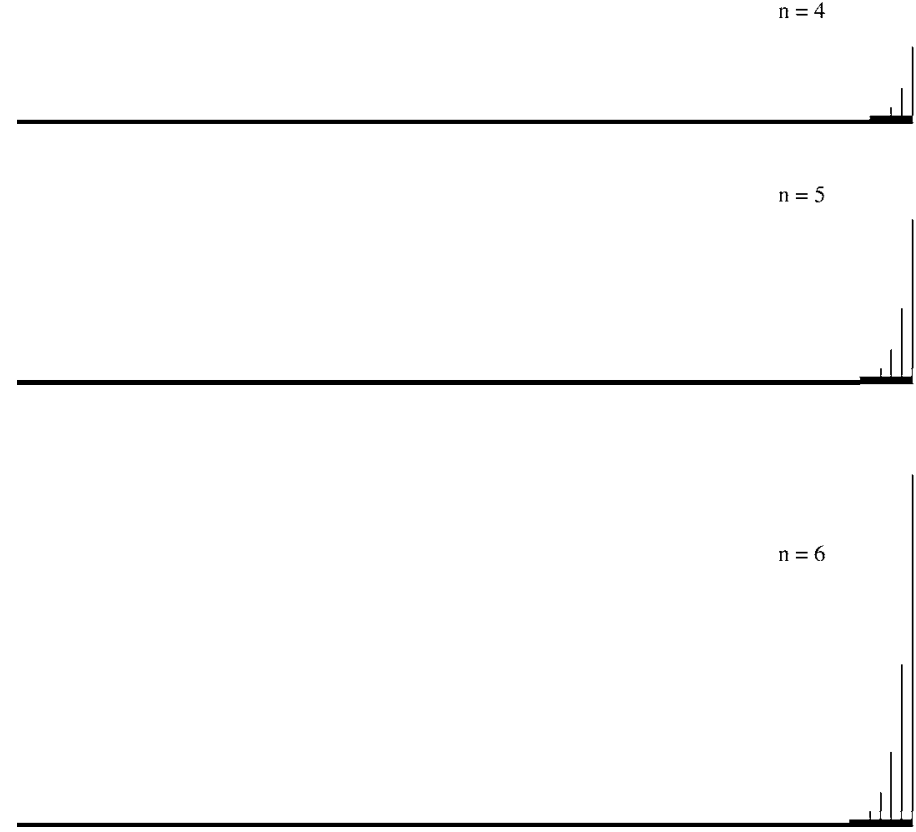

Figure 9. Densities of the binomial multiplicative process for Ce I. The height of the bars is proportional to the density $p_{i} / l_{i}$.

one obtains

\begin{tabular}{llll}
\hline \multicolumn{2}{c}{$\mathrm{Ce}_{\mathrm{I}}$} & \multicolumn{2}{c}{$\mathrm{Ce}_{\mathrm{II}}$} \\
\hline$p_{0}=0.0257$ & $p_{1}=0.9743$ & $p_{0}=0.0289$ & $p_{1}=0.9711$ \\
$l_{0}=0.0120$ & $l_{1}=0.9880$ & $l_{0}=0.0144$ & $l_{1}=0.9856$ \\
\hline
\end{tabular}

Note that $p_{0}$ and $l_{0}$ are very small.

Using these probabilities and lengths in the following equation:

$$
p_{0}^{q} l_{0}^{\tau(q)}+p_{1}^{q} l_{1}^{\tau(q)}=1
$$

allows the sequence of mass exponents $\tau(q)$ to be solved for numerically. A comparison of the 'experimental' $\tau(q)$ and those found 'numerically' from equation (8) is shown in figure 7 . For both $\mathrm{Ce}_{\mathrm{I}}$ and $\mathrm{Ce}$ II the $\tau(q)$ agree well for $q \geqslant-2$ but start to deviate from one another below $q=-2$.

The $f(\alpha)$ spectra were then calculated and are compared with the 'experimental' results as shown in figure 8. There is also deviation of the experimental and numerical $f(\alpha)$ spectra.

In order to compare the experimental and numerical $f(\alpha)$ spectra, $q$ in the range $-10 \leqslant q \leqslant 185$ and $-10 \leqslant q \leqslant 193$ were used for the numerical $f(\alpha)$ spectra of $\mathrm{Ce}_{\mathrm{I}}$ and $\mathrm{Ce}$ II, respectively.

The deviations of the $f(\alpha)$ comparisons occur for negative $q$, i.e. for the low-density regions of the energy eigenvalue sequences. The negative $q$ are much more sensitive in these cases because of the limited number of data points used to generate the 'experimental' $\tau(q)$. The close correspondence between the experimental and numerical $f(\alpha)$ spectra for 

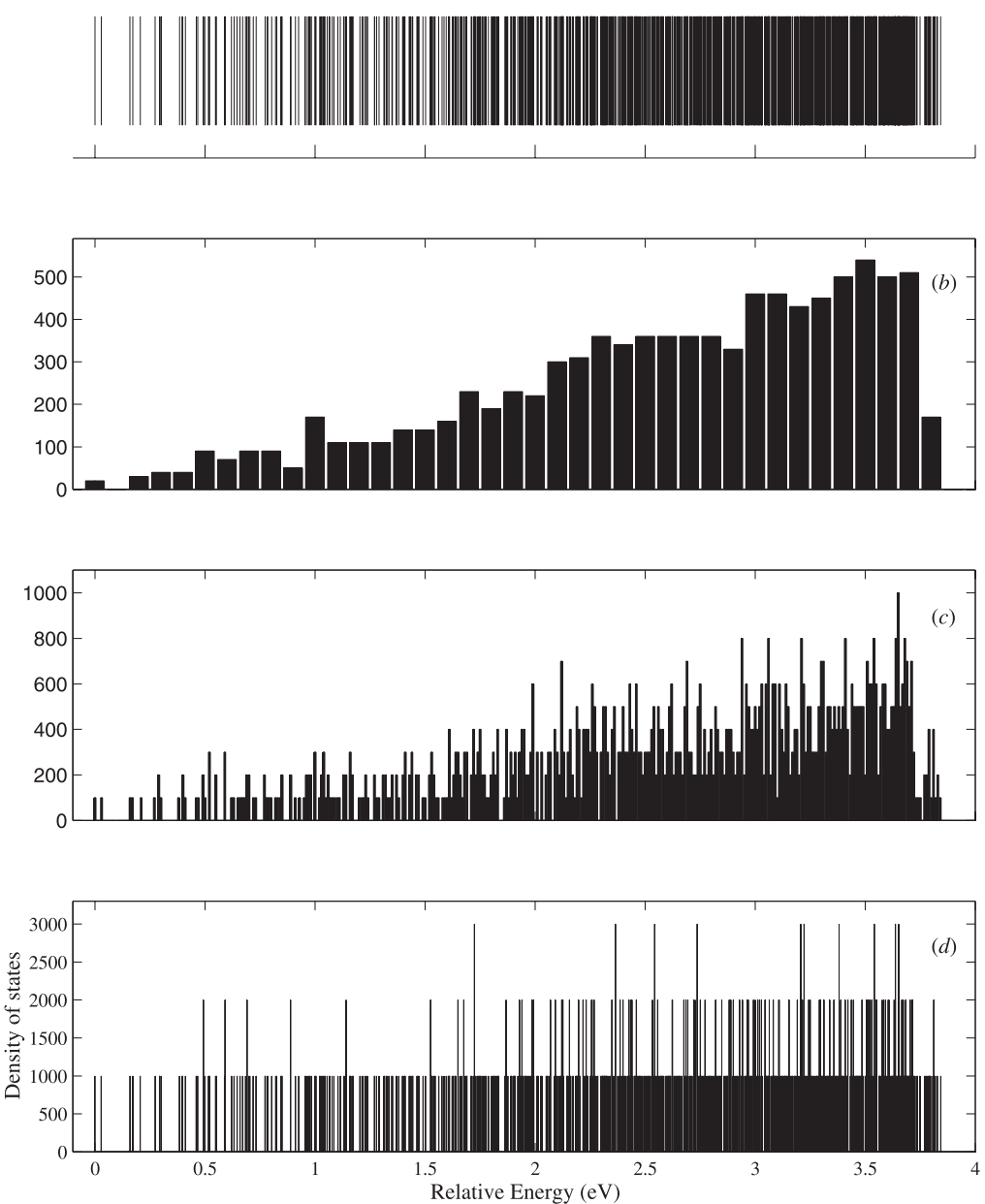

Figure 10. The density of states for CeI: (a) eigenvalue spectrum; (b) $\Delta E=0.1 \mathrm{eV}$; (c) $\Delta E=0.01 \mathrm{eV}$; (d) $\Delta E=0.001 \mathrm{eV}$.

positive $q$ does not mean that the underlying probabilities and lengths used for the binomial multiplicative process are in any way related to the processes that gave rise to the $\mathrm{Ce}_{\mathrm{I}}$ and Ce II experimental $f(\alpha)$ spectra. The $f(\alpha)$ spectra describe the universality of the various scaling exponents that a distribution may have. Why the $f(\alpha)$ spectrum of a two-scale Cantor set should be in close correspondence to the $f(\alpha)$ spectra of $\mathrm{Ce}_{\mathrm{I}}$ and $\mathrm{Ce}$ II is as yet unknown.

Several generations of the multiplicative process for the preceding probabilities and lengths were calculated and are shown as densities $p_{i} / l_{i}$ for generations $n=4$ to 6 in figure 9 for $\mathrm{Ce}$ I. For these very early generations there is a continuum with a small region of high densities. It can be seen that as $n$ increases the region of high density is increasing, although slowly (since $p_{0}$ and $l_{0}$ are very small), so as $n \rightarrow \infty$ one would expect 'spikes' of high density 'sitting on' a continuous background. 

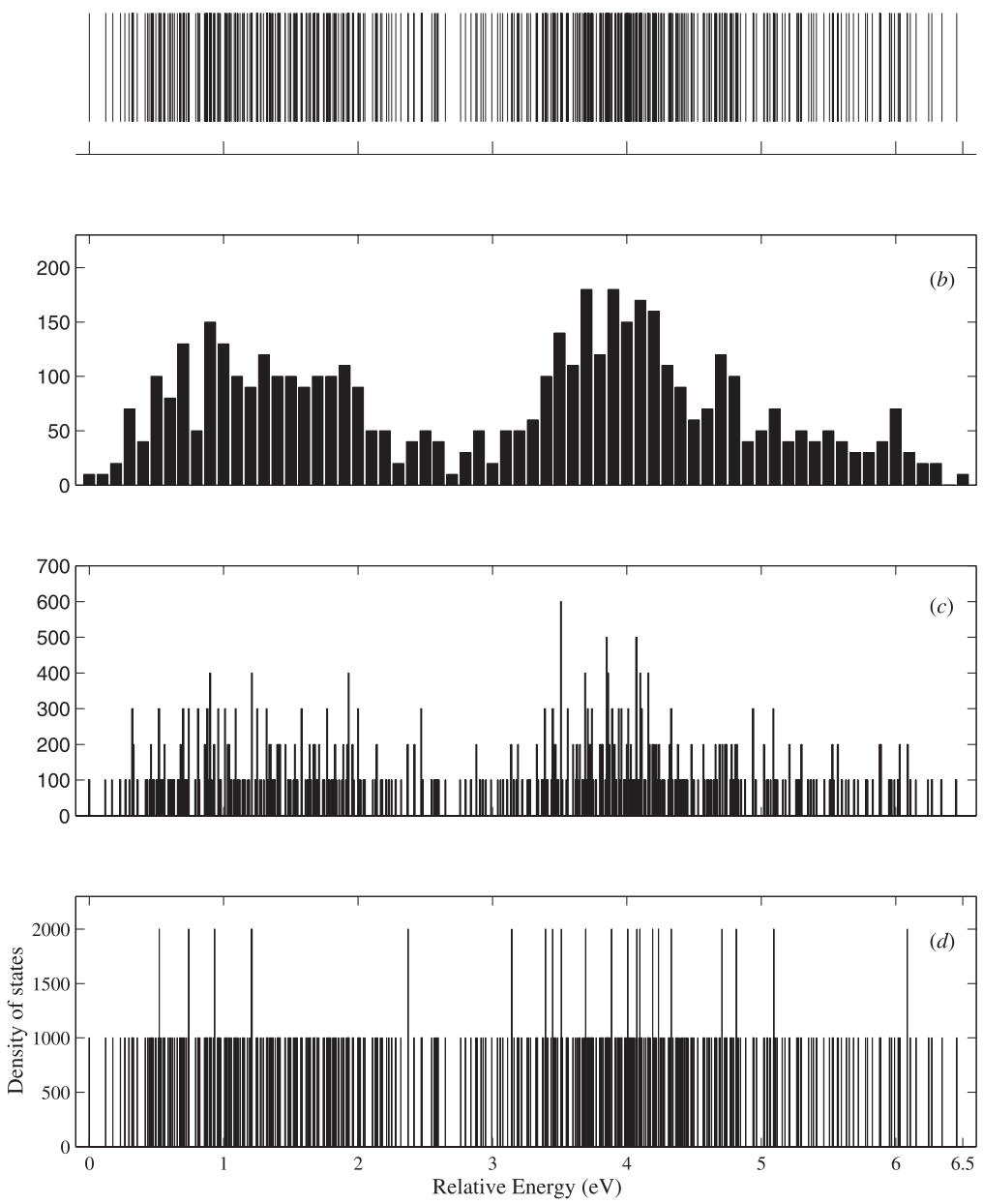

Figure 11. The density of states for Ce II: (a) eigenvalue spectrum; (b) $\Delta E=0.1 \mathrm{eV}$; (c) $\Delta E=0.01 \mathrm{eV} ;(d) \Delta E=0.001 \mathrm{eV}$.

To see if this corresponds to the experimental energies, the level densities- the number of energy levels per unit energy interval-were calculated for intervals $\Delta E$ of $0.1,0.01$ and $0.001 \mathrm{eV}$. In figure $10(b)$ the exponential rise in the density of states as energy increases is seen for $\mathrm{Ce}_{\mathrm{I}}$. As the energy interval decreases there are 'spikes' of high density on a 'nearly' continuous background. When the energy level approaches zero as with $0.001 \mathrm{eV}$ the density of states becomes similar to the eigenvalue spectrum itself_-as shown in figures $10(a)$ and $(d)$. Of course in the limit $\Delta E \rightarrow 0$ the density of states would form a set of Dirac $\delta$-functions. A similar occurrence of high density regions is seen to occur for Ce II as shown in figure 11. It should be noted that the higher density regions shift in energy as $\Delta E$ decreases because of the differing locations of the energy intervals as $\Delta E$ decreases.

Subsets, which are in this case energy intervals (for a given $\Delta E$ ), that have level densities that are the same or are of the same order, correspond to subsets with the same scaling exponent 

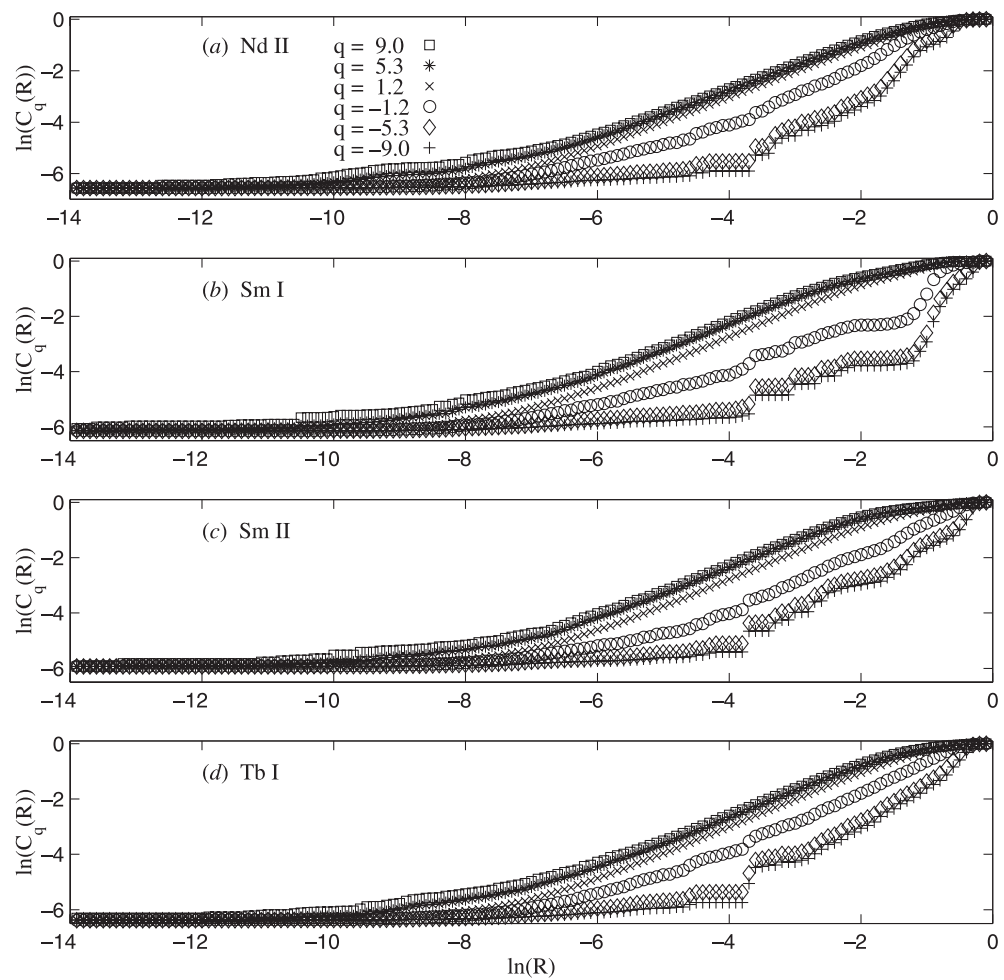

Figure 12. $\operatorname{Ln}\left(C_{q}(R)\right)$ versus $\ln (R)$ for: $(a) \mathrm{Nd}$ II; $(b) \mathrm{Sm} \mathrm{I} ;(c) \mathrm{Sm} \mathrm{II}$; and $(d) \mathrm{Tb}$ I.

$\alpha$. Also note that the high density 'spikes' of the multiplicative process decrease in intensity exponentially, even as $n \rightarrow \infty$.

\section{Analysis of Nd, Sm and Tb}

Having applied the multifractal formalism to $\mathrm{Ce}$ I and $\mathrm{Ce}$ II, the generalized correlation sum method was performed on neodymium $\mathrm{Nd}$, samarium $\mathrm{Sm}$ and terbium $\mathrm{Tb}$, these also being members of the rare-earth elements. The main purpose of this was to search for some universality in overall behaviour, which might mirror the fact that the chemical and physical properties of these elements are so similar due to the very effective screening of the additional nuclear charge by addition of a highly localized $4 \mathrm{f}$ electron to the core in progressing along the sequence. Any 'glitches' that occur can be traced to valence changes and can be understood in terms of half filled or completed $4 \mathrm{f}$ subshells. The number of energy eigenvalues and range of energies used (relative to the ground state) are shown in table 3.

The even and odd configurations that give rise to these eigenvalues are shown in table 4 . Following the same procedure as that used for $\mathrm{Ce}_{\mathrm{I}}$ and $\mathrm{Ce} \mathrm{II}, \ln \left(C_{q}(R)\right)$ was calculated as a function of $\ln (R)$, with the results shown in figure 12 for Nd II, Sm I, Sm II and Tb I. The scaling regions are shown in table 5. Note the larger scaling regions for Sm II and $\mathrm{Tb}$.

Figures 13-16 show the generalized dimensions $D_{q}$ and $\tau(q)$. Note the large error of $D_{q}$, for negative $q$, for Sm I in figure 14(a). This large error is predominantly caused by the small region of scaling that is used to calculate $D_{q}$. However, for all the elements $\mathrm{Ce}, \mathrm{Nd}, \mathrm{Sm}$ and Tb the negative $q$ give large percentage errors of the order of 5-10\%. Calculating the Legendre 

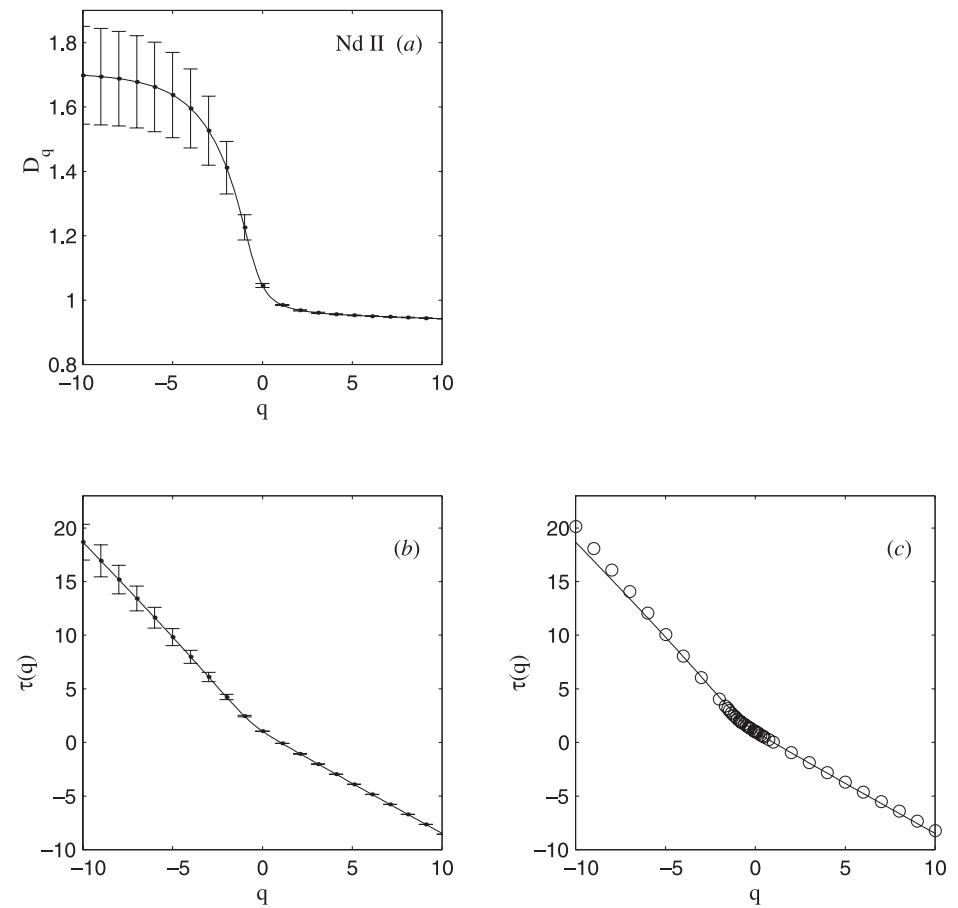

Figure 13. (a) Generalized dimensions $D_{q}$; (b) sequence of mass exponents; $(c)$ 'experimental' (full curve) and 'numerical' (circles) $\tau(q)$ for $\mathrm{Nd}$ II.

transforms results in the $f(\alpha)$ spectra as shown in figure 17. The corresponding error limits of $f(\alpha)$ and $\alpha$ are also shown.

A comparison of the $D_{q}$ dimensions and the $f(\alpha)$ spectra for Ce I, Ce II, Nd II, Sm I, Sm II and $\mathrm{Tb}$ I are shown in figures 19 and 20. It can be seen in figure 19 that all the $D_{q}$ for positive $q$ have similar values, however, there appear to be two separate 'bands' appearing in the negative $q$ regions: Sm II and Tb I appear to form a separate 'band'. These 'bands' can also be discerned in the $f(\alpha)$ spectra for the larger $\alpha$ values-the $f(\alpha)$ distribution corresponding to the less dense regions also seem to have two 'bands', with Sm II and Tb I both being in the same band. However, these so-called 'bands' may have their origin in the fact that only a restricted number of energy eigenvalues were available for analysis. Also, observe that the error in $D_{q}$ for each atom and/or ion can account for and so negate this apparent bandedness. It can also be seen that the $f(\alpha)$ spectra of Sm II and Tb I have a smaller range of Lipschitz-Hölder exponents than $\mathrm{Ce}$ I, Ce II, Nd II and Sm I, suggesting a smaller class of scaling subsets within each set of energy eigenvalues. The $f(\alpha)$ spectra coincide quite closely for positive $q$ and this suggests that all

Table 3. Number of energy eigenvalues and their relative energy range for $\mathrm{Nd}, \mathrm{Sm}$ and $\mathrm{Tb}$.

\begin{tabular}{lll}
\hline & Number of energy eigenvalues & Relative energy (eV) \\
\hline $\mathrm{Nd}_{\text {II }} Z=60$ & 706 & $0-5.830$ \\
$\mathrm{Sm}_{\text {I }} Z=62$ & 469 & $0-4.361$ \\
$\mathrm{Sm}_{\text {II }} Z=62$ & 376 & $0-4.774$ \\
$\mathrm{~Tb}_{\text {I }} Z=65$ & 590 & $0-4.339$ \\
\hline
\end{tabular}



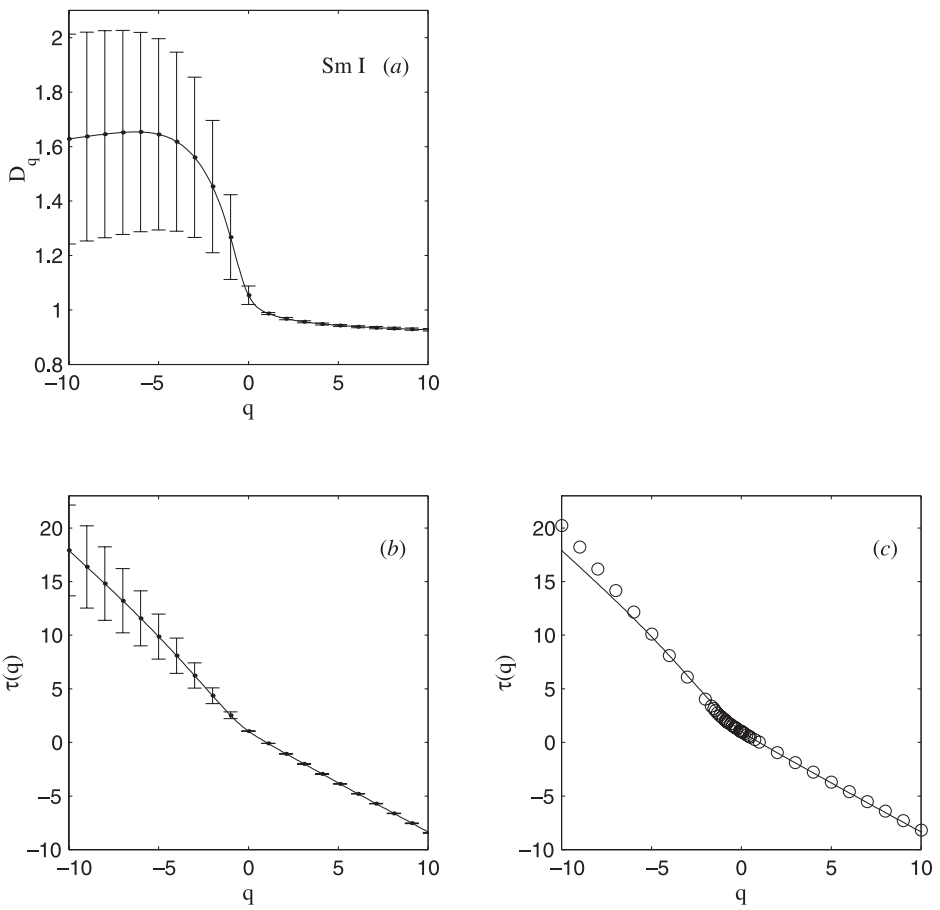

Figure 14. (a) Generalized dimensions $D_{q}$; (b) sequence of mass exponents $\tau(q)$; (c) 'experimental' (full curve) and 'numerical' (circles) $\tau(q)$ for Sm I.

Table 4. Even and odd configurations of Nd II, Sm I, Sm II and Tb I.

\begin{tabular}{ll}
\hline Nd II & \\
Ground configuration & $1 \mathrm{~s}^{2} 2 \mathrm{~s}^{2} 2 \mathrm{p}^{6} 3 \mathrm{~s}^{2} 3 \mathrm{p}^{6} 3 \mathrm{~d}^{10} 4 \mathrm{~s}^{2} 4 \mathrm{p}^{6} 4 \mathrm{~d}^{10} 5 \mathrm{~s}^{2} 5 \mathrm{p}^{6} 4 \mathrm{f}^{4} 6 \mathrm{~s}$ \\
Odd configurations & $4 \mathrm{f}^{3} 5 \mathrm{~d}^{2}, 4 \mathrm{f}^{3} 5 \mathrm{~d} 6 \mathrm{~s}, 4 \mathrm{f}^{4} 6 \mathrm{p}$ \\
Even configurations & $4 \mathrm{f}^{4} 6 \mathrm{~s}, 4 \mathrm{f}^{4} 5 \mathrm{~d}, 4 \mathrm{f}^{3} 5 \mathrm{~d} 6 \mathrm{p}$ \\
Sm I & \\
Ground configuration & $1 \mathrm{~s}^{2} 2 \mathrm{~s}^{2} 2 \mathrm{p}^{6} 3 \mathrm{~s}^{2} 3 \mathrm{p}^{6} 3 \mathrm{~d}^{10} 4 \mathrm{~s}^{2} 4 \mathrm{p}^{6} 4 \mathrm{~d}^{10} 5 \mathrm{~s}^{2} 5 \mathrm{p}^{6} 4 \mathrm{f}^{6} 6 \mathrm{~s}^{2}$ \\
Odd configurations & $4 \mathrm{f}^{6} 6 \mathrm{~s} 6 \mathrm{p}, 4 \mathrm{f}^{5} 5 \mathrm{~d} 6 \mathrm{~s}^{2}, 4 \mathrm{f}^{5} 5 \mathrm{~d}^{2} 6 \mathrm{~s}, 4 \mathrm{f}^{6} 5 \mathrm{~d} 6 \mathrm{p}$ \\
Even configurations & $4 \mathrm{f}^{6} 6 \mathrm{~s}^{2}, 4 \mathrm{f}^{6} 5 \mathrm{~d} 6 \mathrm{~s}, 4 \mathrm{f}^{6} 6 \mathrm{~s} 7 \mathrm{~s}$ \\
Sm II & \\
Ground configuration & $1 \mathrm{~s}^{2} 2 \mathrm{~s}^{2} 2 \mathrm{p}^{6} 3 \mathrm{~s}^{2} 3 \mathrm{p}^{6} 3 \mathrm{~d}^{10} 4 \mathrm{~s}^{2} 4 \mathrm{p}^{6} 4 \mathrm{~d}^{10} 5 \mathrm{~s}^{2} 5 \mathrm{p}^{6} 4 \mathrm{f}^{6} 6 \mathrm{~s}$ \\
Odd configurations & $4 \mathrm{f}^{7}, 4 \mathrm{f}^{6} 6 \mathrm{p}, 4 \mathrm{f}^{5} 5 \mathrm{~d} 6 \mathrm{~s}$ \\
Even configurations & $4 \mathrm{f}^{6} 6 \mathrm{~s}, 4 \mathrm{f}^{6} 5 \mathrm{~d}$ \\
Tb I & \\
Ground configuration & $1 \mathrm{~s}^{2} 2 \mathrm{~s}^{2} 2 \mathrm{p}^{6} 3 \mathrm{~s}^{2} 3 \mathrm{p}^{6} 3 \mathrm{~d}^{10} 4 \mathrm{~s}^{2} 4 \mathrm{p}^{6} 4 \mathrm{~d}^{10} 5 \mathrm{~s}^{2} 5 \mathrm{p}^{6} 4 \mathrm{f}^{9} 6 \mathrm{~s}^{2}$ \\
Odd configurations & $4 \mathrm{f}^{9} 6 \mathrm{~s}^{2}, 4 \mathrm{f}^{9} 5 \mathrm{~d} 6 \mathrm{~s}, 4 \mathrm{f}^{8} 6 \mathrm{~s}^{2} 6 \mathrm{p}, 4 \mathrm{f}^{8} 5 \mathrm{~d} 6 \mathrm{~s} 6 \mathrm{p}, 4 \mathrm{f}^{8} 5 \mathrm{~d}^{2} 6 \mathrm{p}, 4 \mathrm{f}^{9} 6 \mathrm{~s} 7 \mathrm{~s}$ \\
Even configurations & $4 \mathrm{f}^{8} 5 \mathrm{~d} 6 \mathrm{~s}^{2}, 4 \mathrm{f}^{8} 5 \mathrm{~d}^{2} 6 \mathrm{~s}, 4 \mathrm{f}^{9} 6 \mathrm{~s} 6 \mathrm{p}$
\end{tabular}

the dense regions of the various eigenvalue sequences are similar in energy distribution. In fact, the density of states for $\Delta E=0.1 \mathrm{eV}$, for $\mathrm{Ce}, \mathrm{Nd}, \mathrm{Sm}$ and $\mathrm{Tb}$, all peak in the relative energy region of approximately $3-4.5 \mathrm{eV}$ as can be seen in $(b)$ of figures 10,11 and figures $21-24$. The maximum value of $f(\alpha)$, as well as $D_{0}$, for all the selected atoms and ions is approximately 1.0. 

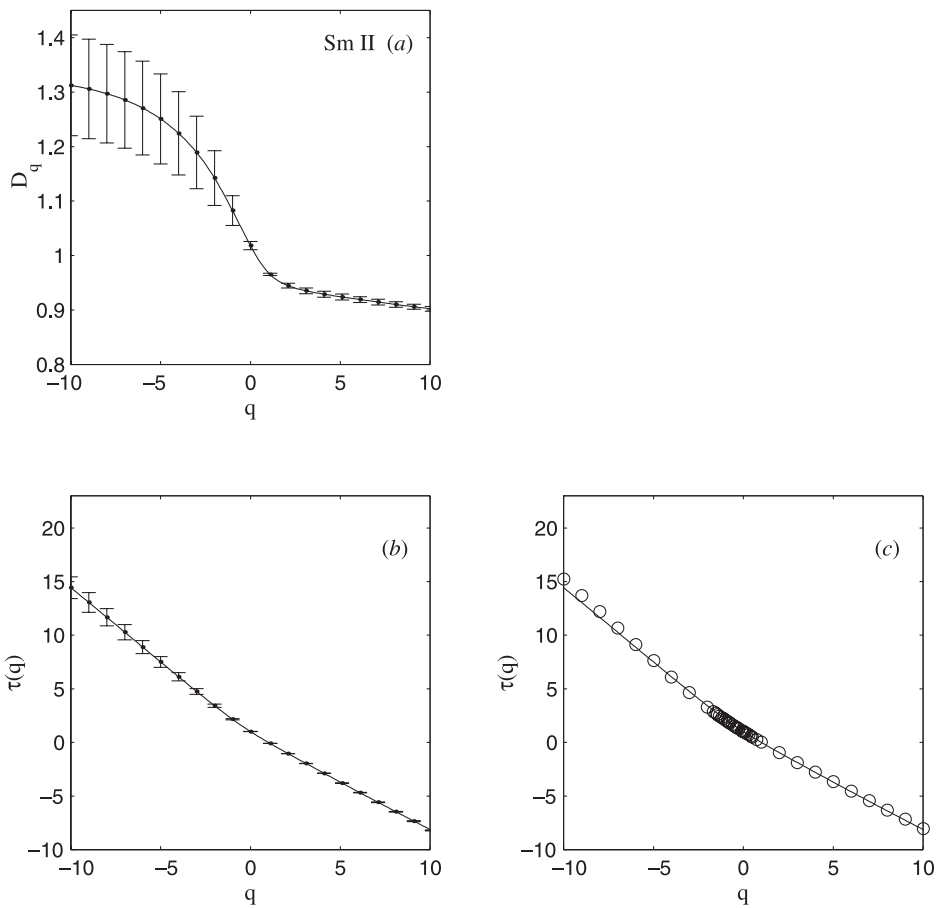

Figure 15. (a) Generalized dimensions $D_{q} ; \quad(b)$ sequence of mass exponents $\tau(q)$ (c) 'experimental' (full curve) and 'numerical' (circles) $\tau(q)$ for Sm II.

This suggests that the support of the measure is a continuum. The Kolmogorov capacity $D_{0}$, information dimension $D_{1}$, correlation dimension $D_{2}$; range of $q$ values used in the Legendre transformations; the maximum and minimum values of the Lipschitz-Hölder exponents $\alpha$ and the maximum value of $f(\alpha)$ are shown in table 5, for $\mathrm{Nd}$ II, Sm I, Sm II and Tb I.

As with $\mathrm{Ce}_{\mathrm{I}}$ and $\mathrm{Ce}$ II, the $f(\alpha)$ spectra for a binomial multiplicative process with a continuous support for the measure was calculated numerically, in order to try to simulate the $f(\alpha)$ spectra. The resulting $f(\alpha)$ distributions are shown and compared with the 'experimental' $f(\alpha)$ distributions in figure 18. The positive $q$ regions for both the 'experimental' and 'numerical' $f(\alpha)$ spectra are again similar in magnitude, however, the negative $q$ regions deviate for Nd II, Sm I and Sm II. The coincidence of the $f(\alpha)$ spectra of Tb I for the less dense regions may be purely a numerical artefact. The range of $q$ values used for the numerical $f(\alpha)$ spectra are shown in table 5. The deviation of the negative $q$ regions can also be seen in the 'experimental' and 'numerical' $\tau(q)$, as shown in $(c)$ of figures 13-16.

The density of states for various energy intervals $\Delta E$ was also calculated and these are shown in figures 21-24. Note the 'spikes' of high density on a nearly continuous background as $\Delta E$ decreases.

It should be noted that the $f(\alpha)$ technique was also applied to the theoretical energy eigenvalues, as calculated by the Cowan suite of codes [20], of the present lanthanide atoms and ions. Tentative results indicate the presence of multi-scaling behaviour in the dense regions of the spectra but not in the less dense regions. This is suspected to arise from insufficient electronelectron correlations in the configuration interaction Hartree-Fock (HFCI) code. Thus the $f(\alpha)$ method would appear to be quite sensitive to the presence or absence of electron-electron 

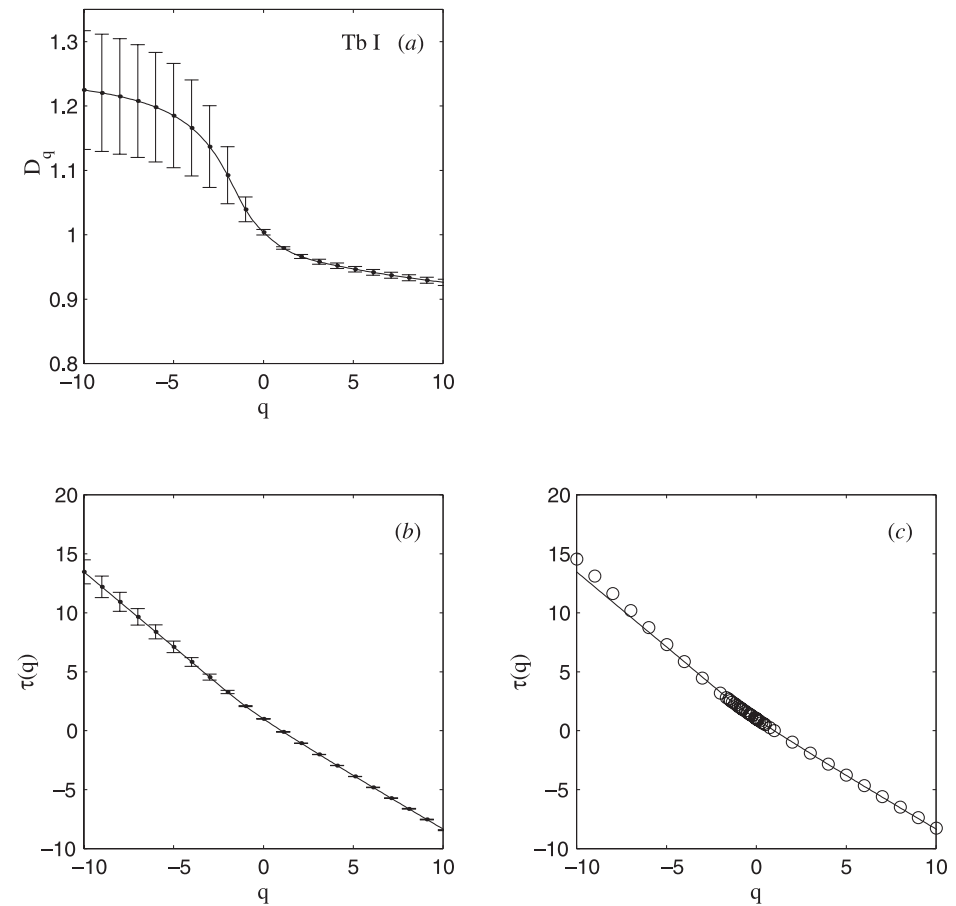

Figure 16. (a) Generalized dimensions $D_{q}$; (b) sequence of mass exponents $\tau(q)$; (c) 'experimental' (full curve) and 'numerical' (circles) $\tau(q)$ for Tb I.

Table 5. Various characteristic parameters for Nd II, Sm I, Sm II and Tb I.

\begin{tabular}{|c|c|c|c|c|}
\hline & $\mathrm{Nd}$ II & $\mathrm{Sm} I$ & Sm II & $\mathrm{Tb}_{\mathrm{I}}$ \\
\hline $\ln (R)$ & $\begin{array}{c}-3.8 \leqslant \ln (R) \leqslant-2.8 \\
0.022 \leqslant R \leqslant 0.061\end{array}$ & $\begin{array}{c}8-3.9 \leqslant \ln (R) \leqslant-3.3 \\
0.020 \leqslant R \leqslant 0.037\end{array}$ & $\begin{array}{c}3.8 \leqslant \ln (R) \leqslant-2.4 \\
0.022 \leqslant R \leqslant 0.091\end{array}$ & $\begin{array}{c}4-4.0 \leqslant \ln (R) \leqslant-2.0 \\
0.018 \leqslant R \leqslant 0.135\end{array}$ \\
\hline$q$ used for $f(\alpha)$ & $-2.8 \leqslant q \leqslant 19.7$ & $-2.4 \leqslant q \leqslant 19.7$ & $-6.0 \leqslant q \leqslant 19.7$ & $-3.2 \leqslant q \leqslant 19.7$ \\
\hline$D_{0}$ & 1.046 & 1.055 & 1.018 & 1.004 \\
\hline$D_{1}$ & 0.987 & 0.990 & 0.969 & 0.981 \\
\hline$D_{2}$ & 0.969 & 0.970 & 0.946 & 0.967 \\
\hline$\alpha_{\min }$ & 0.899 & 0.896 & 0.838 & 0.878 \\
\hline$\alpha_{\max }$ & 1.879 & 1.885 & 1.392 & 1.282 \\
\hline $\max (f(\alpha)) \quad \alpha$ & $1.046 \quad 1.156$ & $1.055 \quad 1.189$ & $1.018 \quad 1.080$ & $1.004 \quad 1.031$ \\
\hline$p_{0}$ & $5.2008 \mathrm{E}-3$ & $6.3742 E-3$ & 0.1424 & 0.1004 \\
\hline$l_{0}$ & $2.5908 \mathrm{E}-3$ & $3.1575 \mathrm{E}-3$ & 0.096 & 0.0702 \\
\hline $\begin{array}{l}q \text { used to } \\
\text { simulate } f(\alpha) \\
\text { spectrum }\end{array}$ & $-10 \leqslant q \leqslant 134$ & $-10 \leqslant q \leqslant 132$ & $-10 \leqslant q \leqslant 62$ & $-10 \leqslant q \leqslant 90$ \\
\hline
\end{tabular}

correlations. In fact, the multifractal experimental results are suspected to arise from complex electron-electron correlations. 

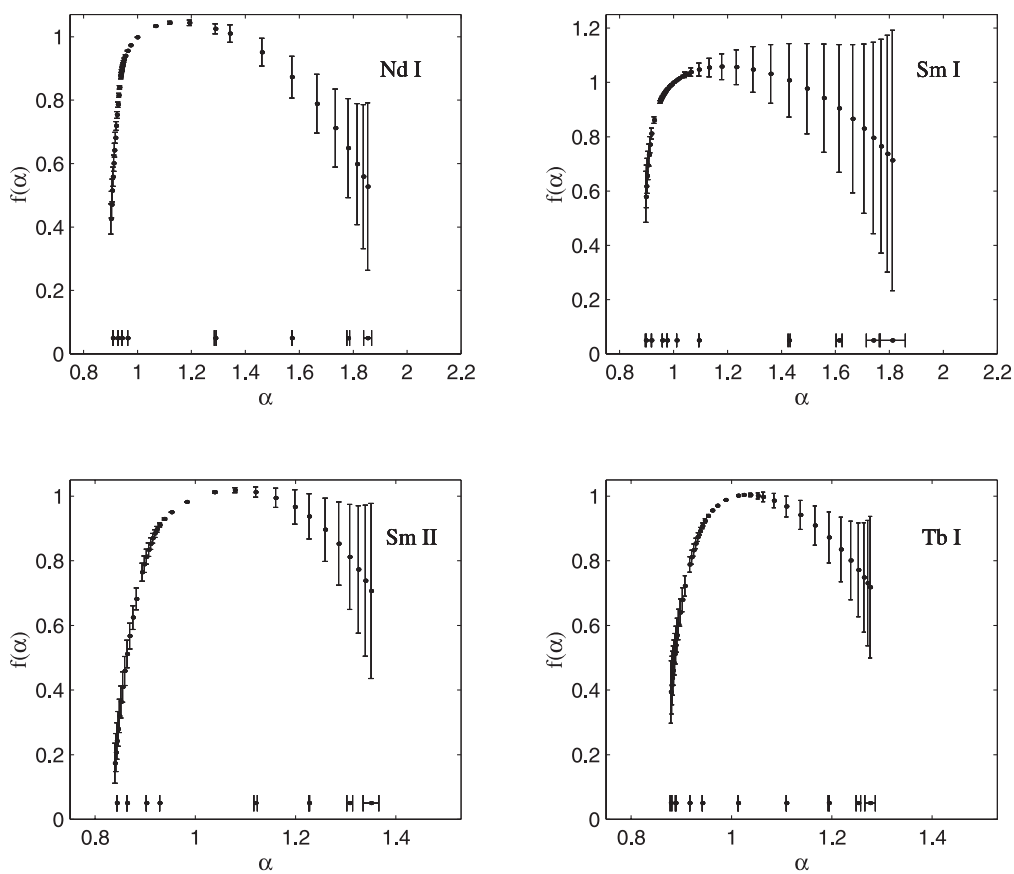

Figure 17. $f(\alpha)$ spectra for Nd II, Sm I, Sm II and Tb I.
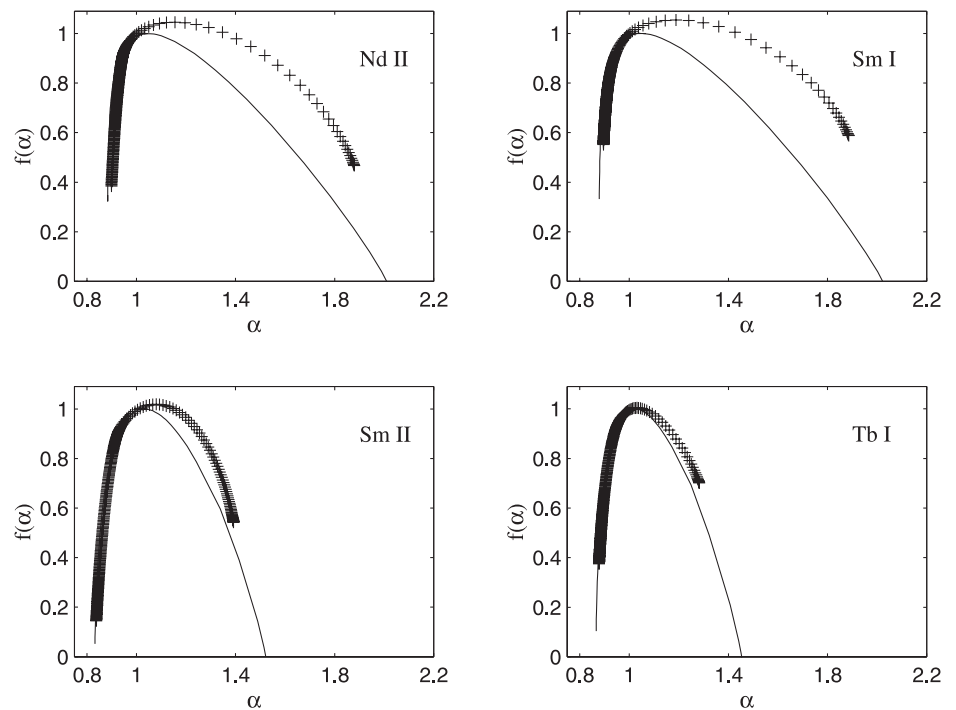

Figure 18. Comparison of 'experimental' (+) and 'numerical' (full) $f(\alpha)$ spectra.

\section{Unfolding and $f(\alpha)$ spectra}

Although all of the experimental $f(\alpha)$ spectra are concave, they do not extend to $f(\alpha)=0$. Also $f\left(\alpha_{\max }\right)$ and $f\left(\alpha_{\min }\right)$ are different in value. This may be an indication of 'some sort' of phase transition. The most obvious correspondence to any phase transition is the variation of 


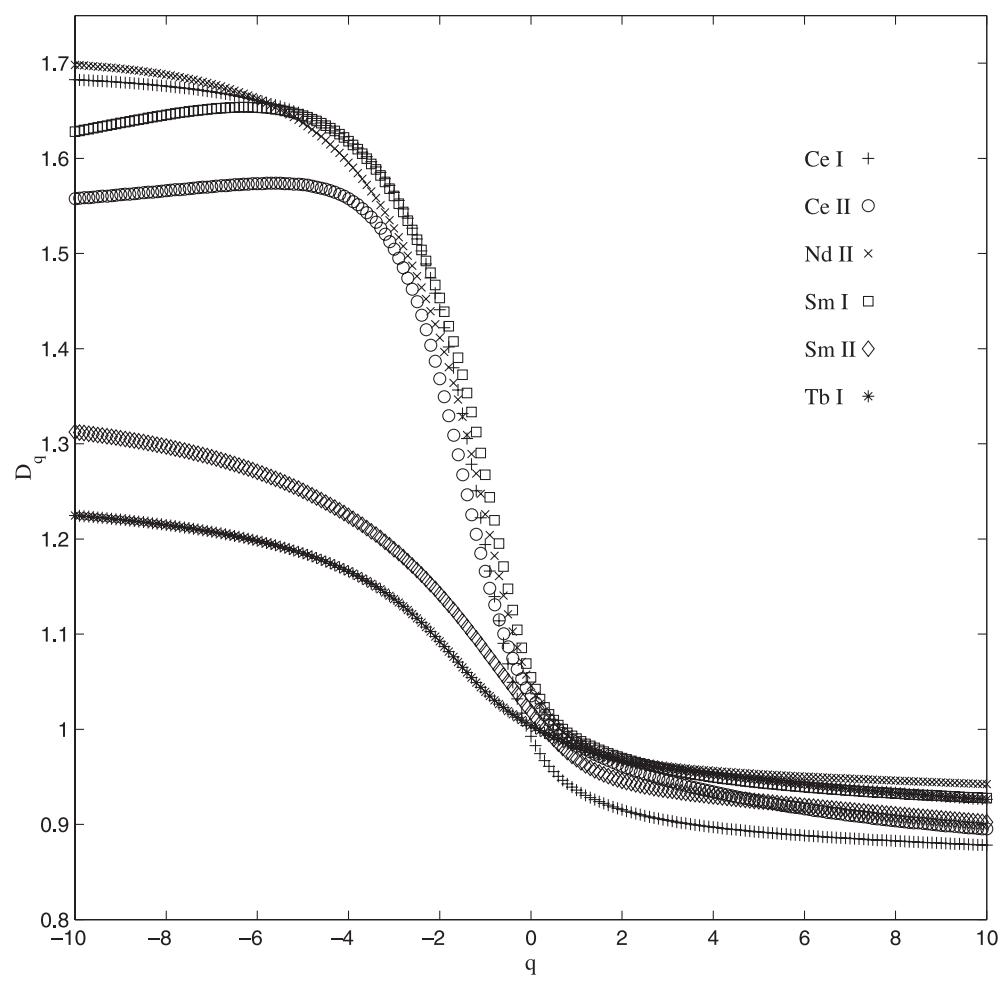

Figure 19. Comparison of the generalized $D_{q}$ dimensions for $\mathrm{Ce}$ I, $\mathrm{Ce}_{\text {II }}, \mathrm{Nd}$ II, $\mathrm{Sm}$ I, Sm II and $\mathrm{Tb}$ I.

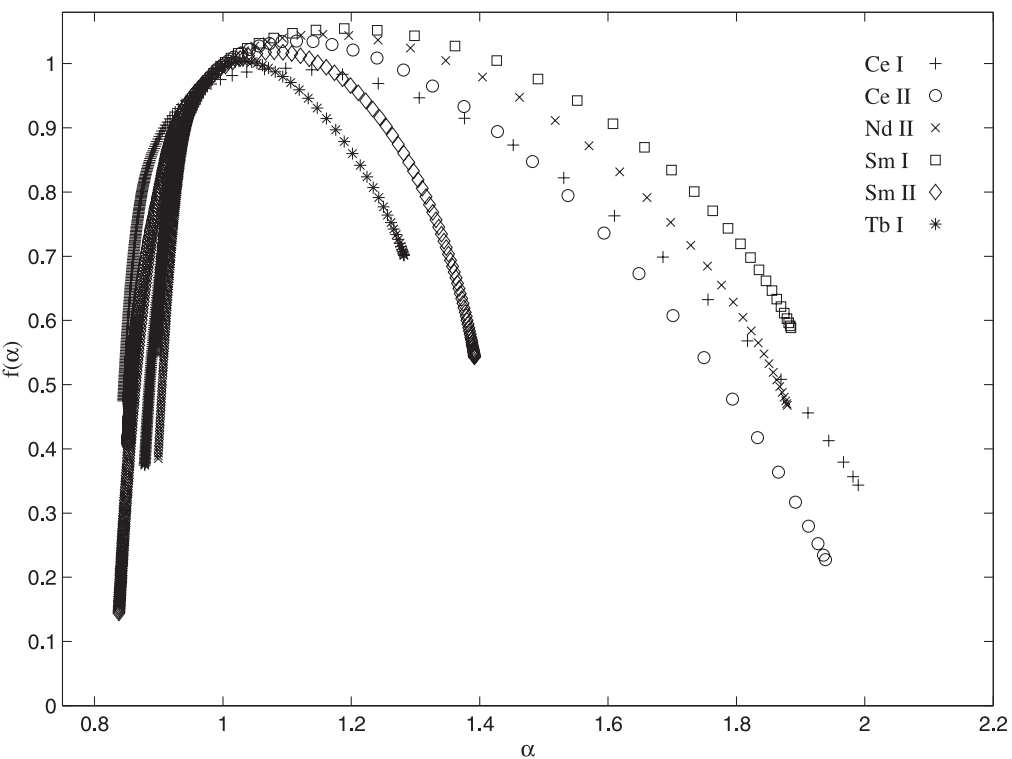

Figure 20. Comparison of the $f(\alpha)$ spectra for $\mathrm{Ce}_{\mathrm{I}}, \mathrm{Ce}_{\text {II, }}, \mathrm{Nd}_{\text {II }}, \mathrm{Sm}_{\mathrm{I}}, \mathrm{Sm}_{\text {II }}$ and $\mathrm{Tb}$ I.

the density of states with changing energy. So to test for the effect that the varying density of 
$\mathrm{Nd}$ II $(a)$
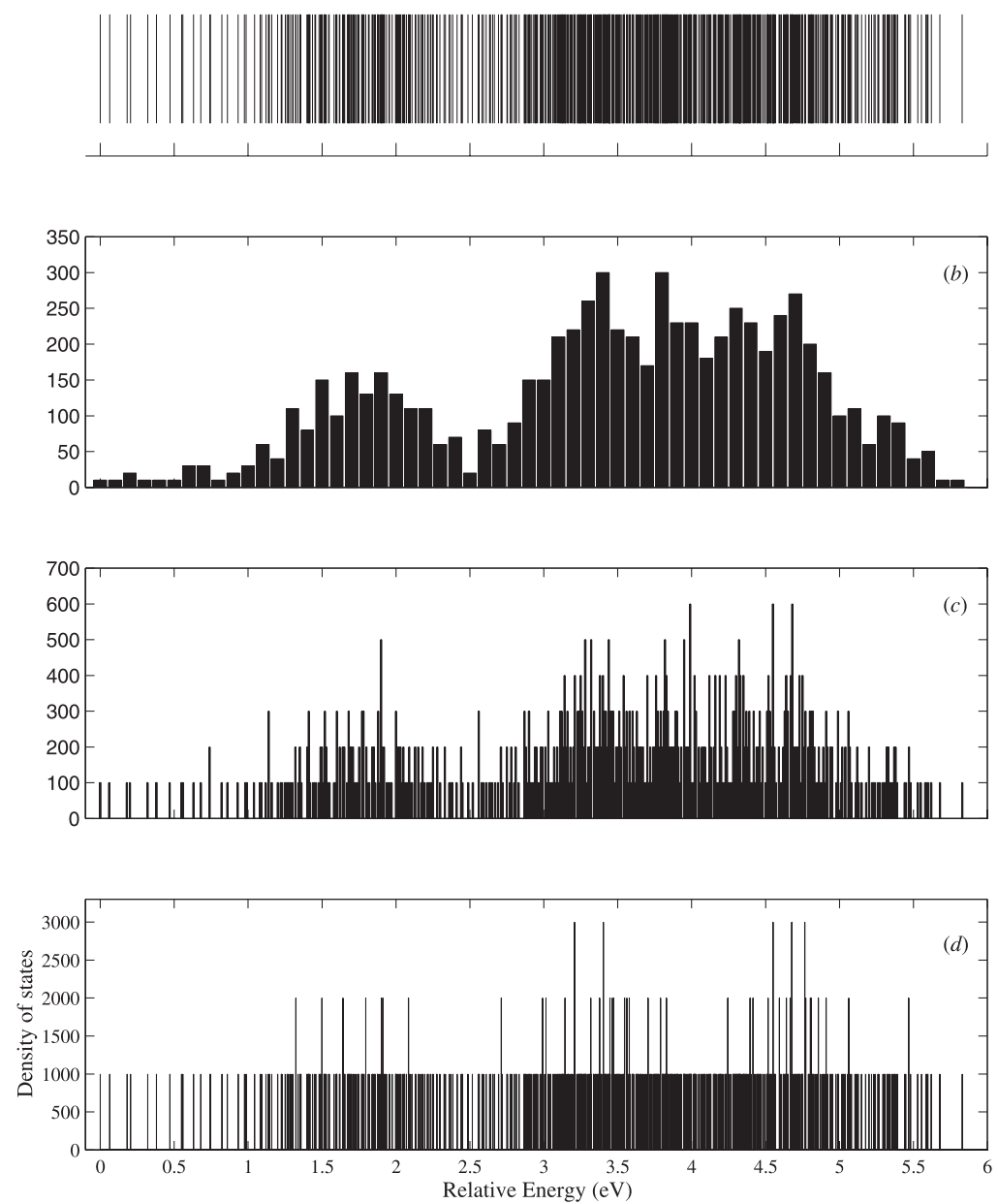

Figure 21. The density of states for $\mathrm{Nd}$ II: $(a)$ eigenvalue spectrum; $(b) \Delta E=0.1 \mathrm{eV}$; (c) $\Delta E=0.01 \mathrm{eV} ;(d) \Delta E=0.001 \mathrm{eV}$.

states might have upon $f(\alpha)$, the Ce I eigenvalues were unfolded [21] using

$$
\bar{N}(E)=\int_{E_{0}}^{E} \rho_{a} \mathrm{~d} E^{\prime}
$$

where $\bar{N}(E)$ is the mean cumulative number of states as a function of energy (mean mode number) and $\rho_{a}$ is the density of states. $E_{0}$ is the minimum energy and $\rho_{a}$ is defined as

$$
\rho_{a}=\rho_{0} \exp \left(a \sqrt{E-E_{0}}\right)
$$

where $\rho_{0}$ and $a$ are treated as curve fitting parameters.

Figure 25(a) shows the mode number and mean mode number for $\mathrm{Ce}$ I. The energy eigenvalues are unfolded by replacing each eigenvalue by the mean mode number at that energy. This results in a mean level density of 1.0 as shown by the broken line of figure $25(b)$. 

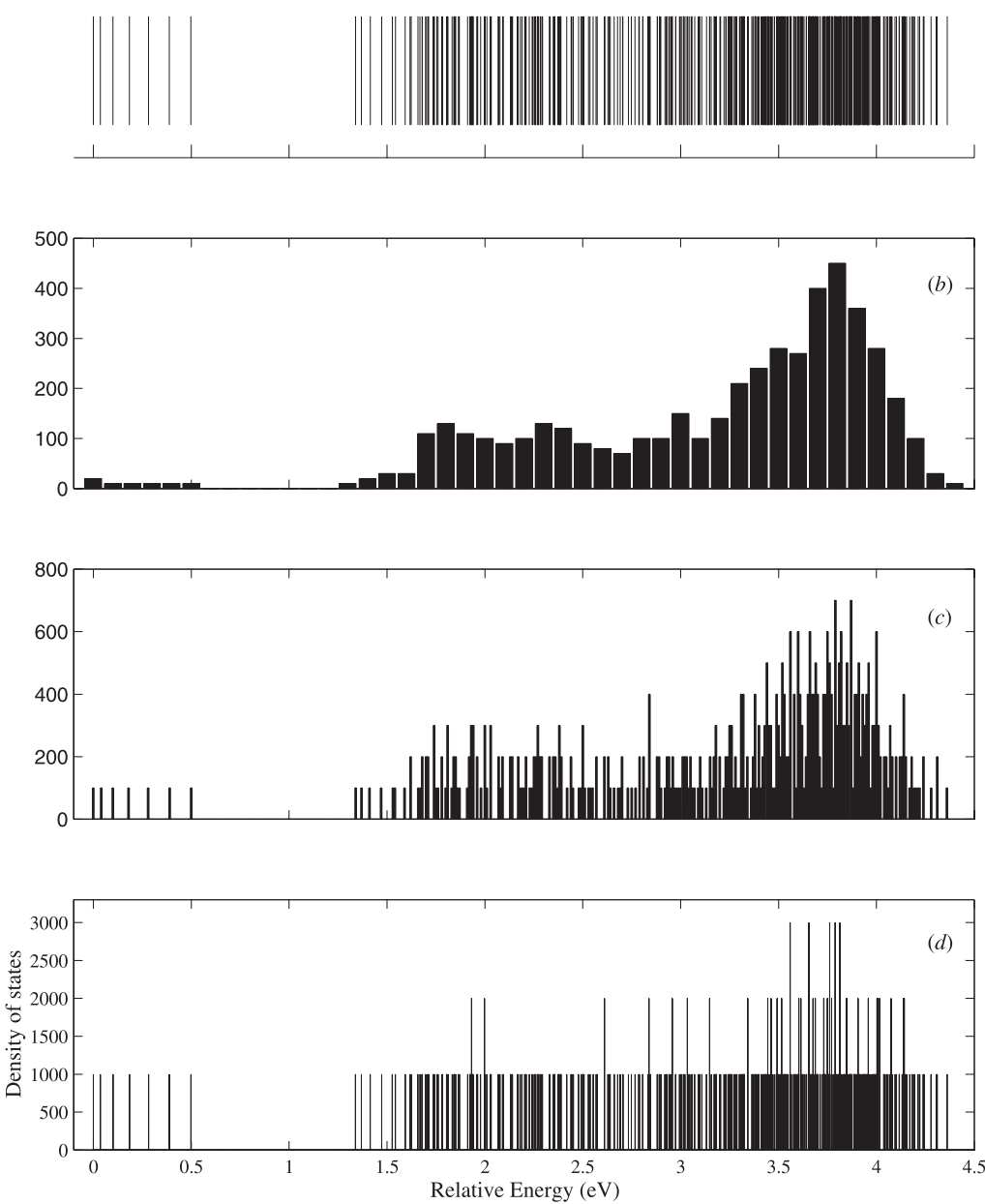

Figure 22. The density of states for SmI: (a) eigenvalue spectrum; (b) $\Delta E=0.1 \mathrm{eV}$; (c) $\Delta E=0.01 \mathrm{eV}$; (d) $\Delta E=0.001 \mathrm{eV}$.

The correlation sum and the resulting $D_{q}, \tau(q)$ and $f(\alpha)$ spectra are determined as before, with results as shown in figures 26-28. The corresponding error limits are also included.

The range of scaling is found to have increased quite dramatically:

$$
-5.0 \leqslant \ln (R) \leqslant-2.0 \equiv 0.0067 \leqslant R \leqslant 0.1353 \quad \text { where } \quad 0.1353 / 0.0067 \approx 20.19 \text {. }
$$

The $f(\alpha)$ spectrum of figure $28(a)$ is now restricted in its $\alpha$ range suggesting a less inhomogenous set of energy levels (see also figure 28(c)). The levels are less inhomogenous in relation to there being a more restricted range of $\alpha$ s. This, however, does not give any indication as to whether the lacunarity [22] has or has not changed.

The minimum values of $f(\alpha)$ are both closer to zero. The maximum value of $f(\alpha)$ is approximately 1.0 as with the pre-unfolded levels. It would be expected that a constant level density of 1.0 would give $\alpha=1.0$ and $f(\alpha)=1.0$, i.e. only one Lipschitz-Hölder exponent. However, the unfolded spectrum still has fluctuations about the mean mode number 

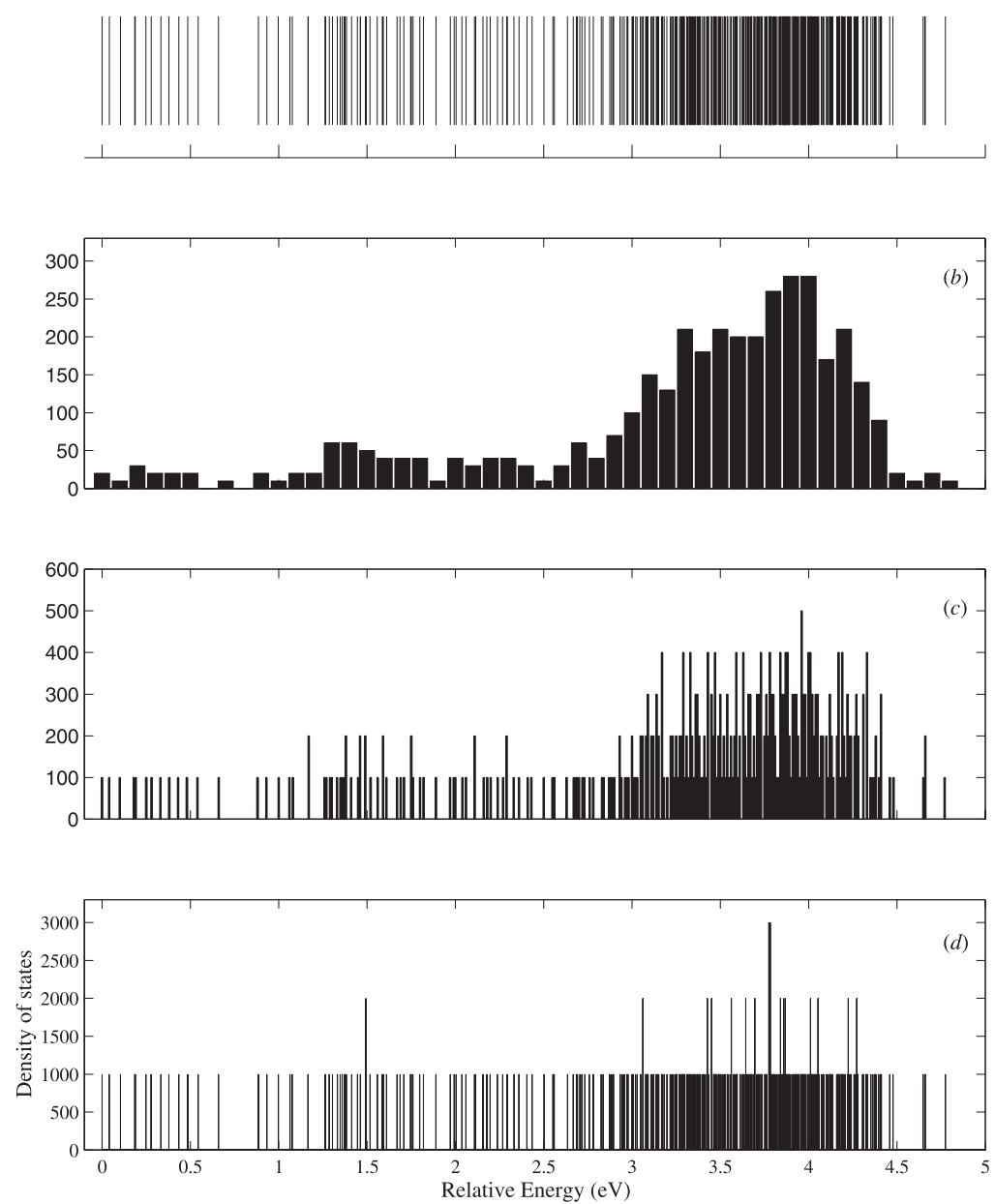

Figure 23. The density of states for Sm II: (a) eigenvalue spectrum; (b) $\Delta E=0.1 \mathrm{eV}$; (c) $\Delta E=0.01 \mathrm{eV} ;(d) \Delta E=0.001 \mathrm{eV}$.

and these give rise to the other $\alpha$ s. Thus, since the $\alpha$ range of the $f(\alpha)$ spectrum is found to be more restricted in extent than that found for the pre-unfolded levels, this would imply that the unfolding (rescaling) process is actually a smoothing procedure.

Table 6 shows the range of $q$ values used in the Legendre transformation, the maximum and minimum values of the Lipschitz-Hölder exponents $\alpha$, as well as the Kolmogorov capacity $D_{0}$, information $D_{1}$ and correlation dimensions $D_{2}$ and the maximum value of $f(\alpha)$.

Again it is interesting to interpolate the $f(\alpha)$ spectrum to find the maximum and minimum $\alpha$ values and then using these values to solve the binomial multiplicative equations to calculate the theoretical sequence of mass exponents $\tau(q)$ for a binomial multiplicative process with a continuous support. The calculated probabilities and lengths are:

$$
\begin{array}{ll}
p_{0}=0.0240 & p_{1}=0.9760 \\
l_{0}=0.0152 & l_{1}=0.9848 .
\end{array}
$$



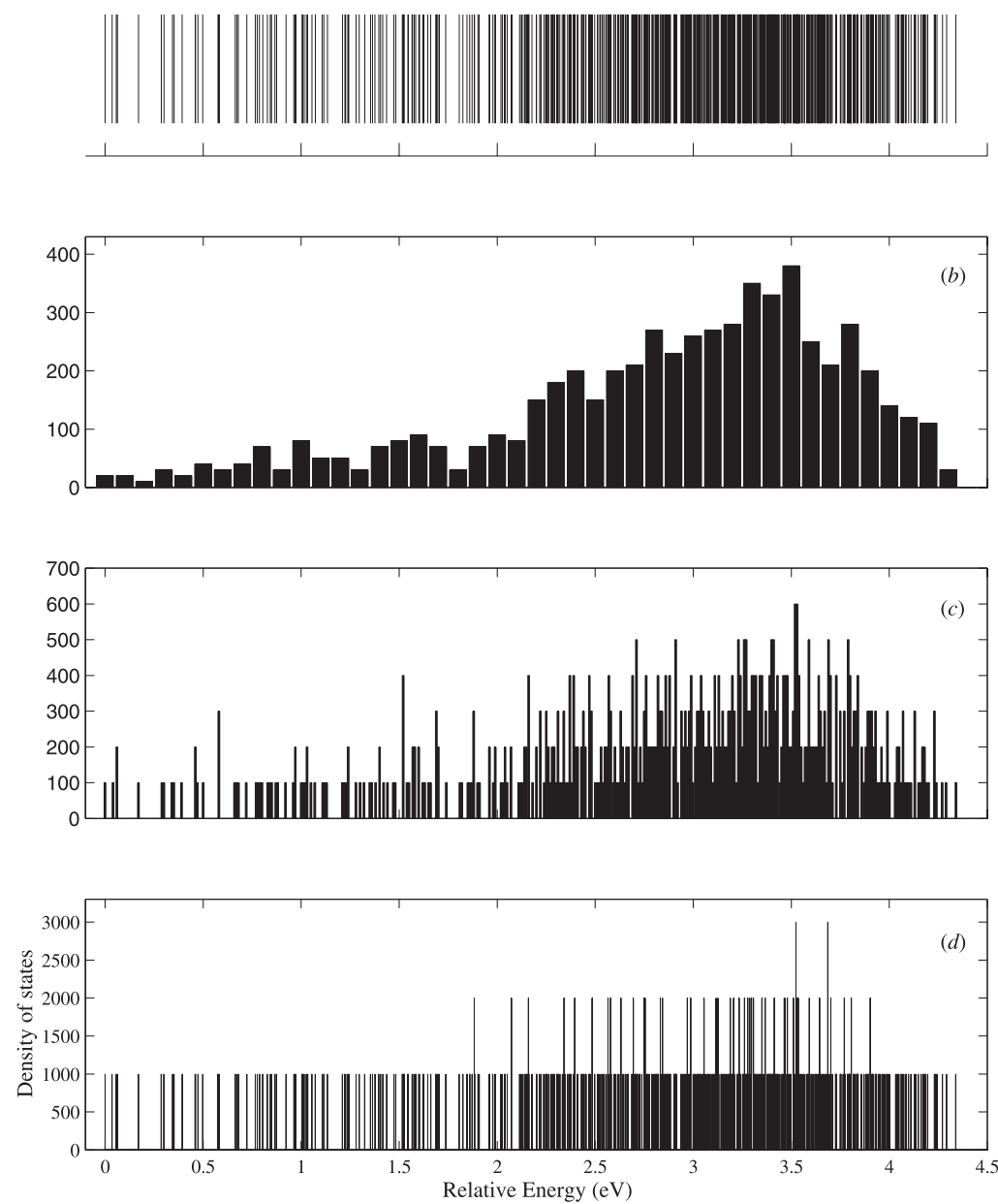

Figure 24. The density of states for TbI: (a) eigenvalue spectrum; (b) $\Delta E=0.1 \mathrm{eV}$; (c) $\Delta E=0.01 \mathrm{eV}$; (d) $\Delta E=0.001 \mathrm{eV}$.

Table 6. Characteristic parameters of unfolded $\mathrm{Ce}$ I.

\begin{tabular}{l}
\hline Unfolded Ce I \\
\hline$-3.0 \leqslant q \leqslant 19.7$ \\
$D_{0}=0.984$ \\
$D_{1}=0.977$ \\
$D_{2}=0.974$ \\
$\alpha_{\min }=0.904$ \\
$\alpha_{\max }=1.53$ \\
$\max (f(\alpha))=0.984 \alpha=0.998$
\end{tabular}

The $\tau(q)$ exponents are shown in figure 27(c) and the resulting $f(\alpha)$ spectrum is shown in figure $28(b)$. It can be seen that there is quite good agreement between the 'experimental' and 

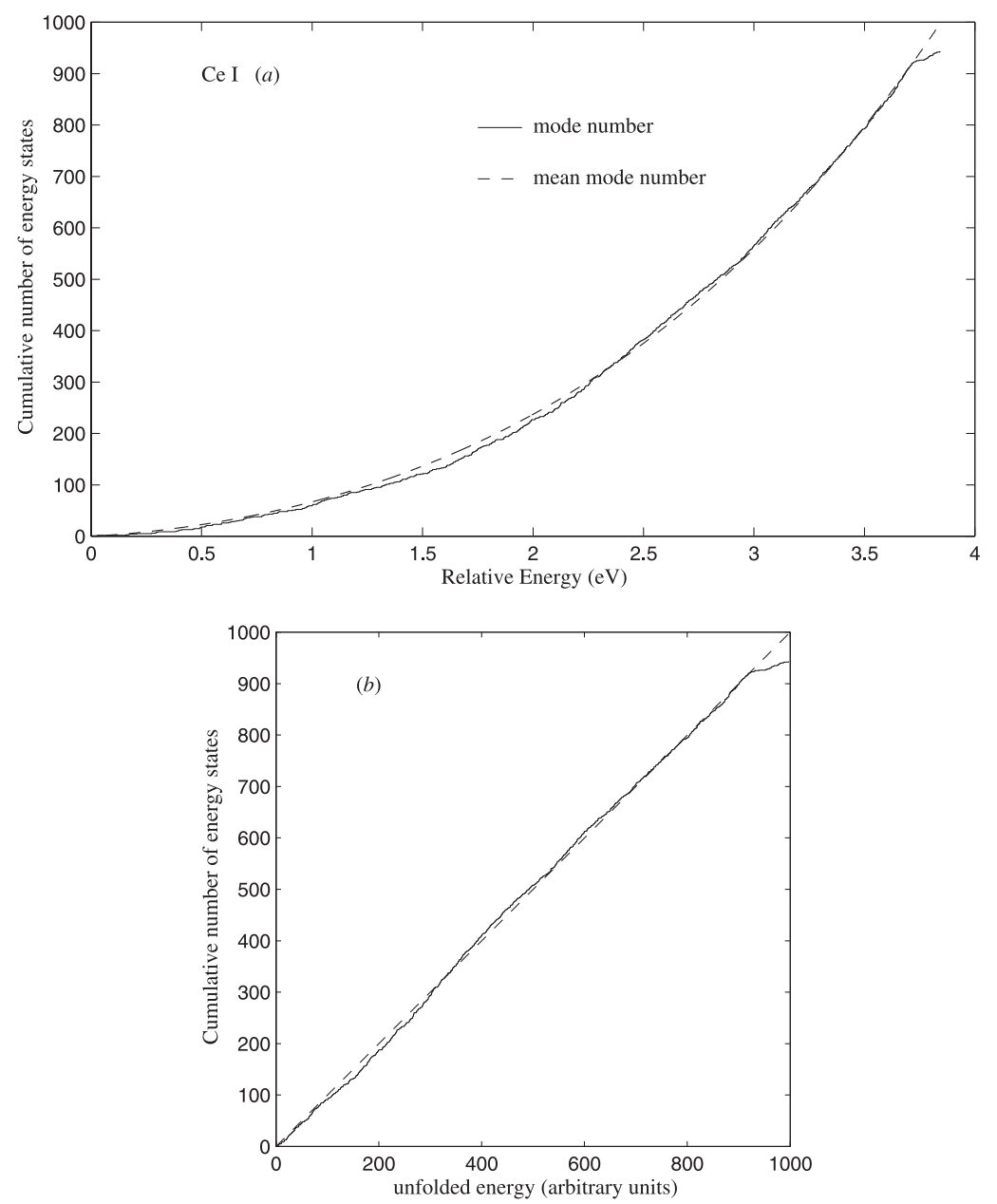

Figure 25. (a) Mode number and mean mode number for $\mathrm{Ce} \mathrm{I} ;(b)$ cumulative number of unfolded energy eigenvalues.

'numerical' $f(\alpha)$ spectra over all the range of $\alpha$. The density of states for the unfolded Ce I is shown in figure 29.

\section{Conclusion}

The multifractal formalism has been applied to sequences of energy eigenvalues for $\mathrm{Ce}_{\mathrm{I}}, \mathrm{Ce}$ II, Nd II, SmI, SmII and Tb I. The resulting Rényi dimensions $D_{q}$, mass exponents $\tau(q)$ and $f(\alpha)$ spectra were calculated with $D_{0} \approx 1.0$ and $\max (f(\alpha)) \approx 1.0$, both suggesting that the energy states are derived from an underlying continuum of energy levels. The fact that $f(\alpha)$ spectra were obtained implies that the energy levels have various subsets with differing scaling exponents $\alpha$, i.e. they are multi-scaling. The $D_{q}$ and $f(\alpha)$ spectra seem to give rise to two 'separate bands' as shown for the negative $q$ regions. These correspond to the less dense regions of the eigenvalue spectra and so may have their origin in the restricted number of eigenvalues that were used in the analysis and/or the limited range of scaling that was 


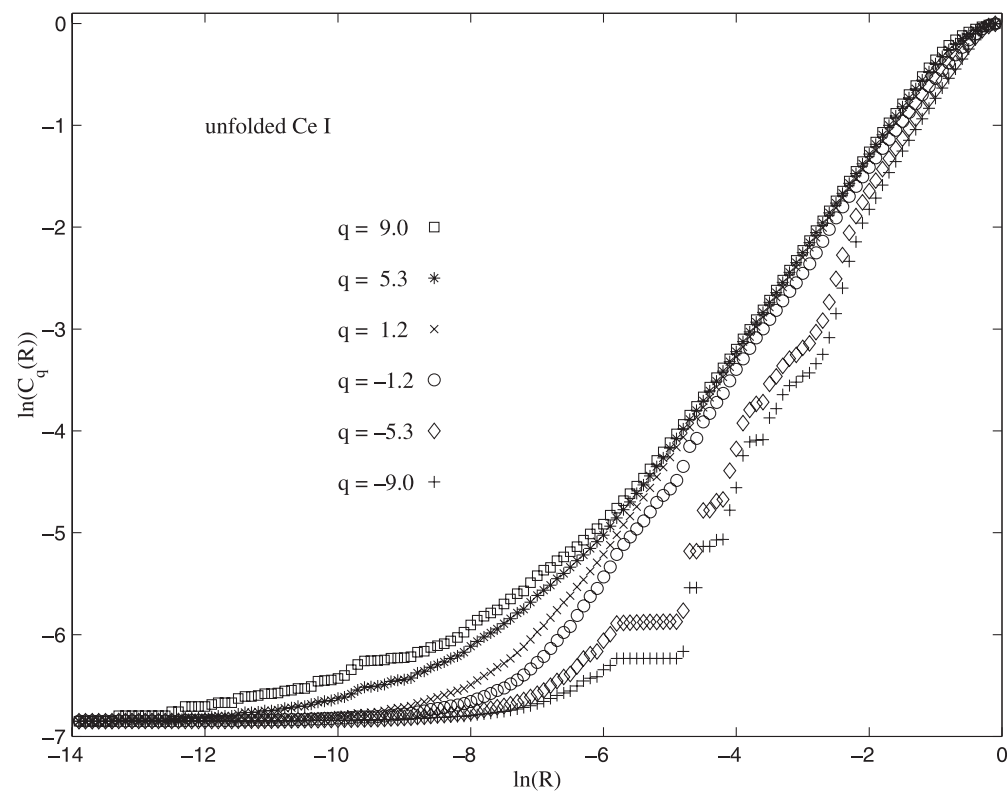

Figure 26. $\operatorname{Ln}\left(C_{q}(R)\right)$ versus $\ln (R)$ for unfolded Ce I.
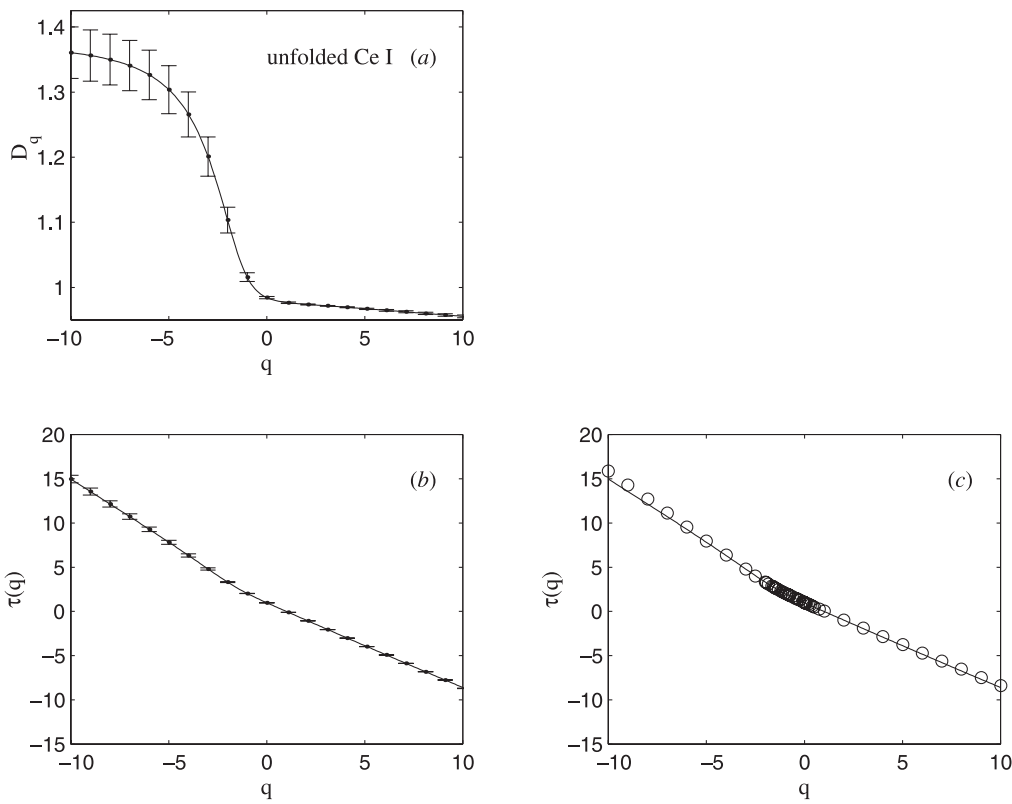

Figure 27. (a) Generalized dimensions $D_{q} ;(b)$ sequence of mass exponents $\tau(q)$; (c) 'experimental' (full curve) and 'numerical' (circles) $\tau(q)$.

obtained. This is confirmed by the corresponding errors in $D_{q}$. However, the more dense regions are not banded and lie very close in value. The $f(\alpha)$ spectra for all the selected atoms and ions are still, however, quite similar in appearance and extend over comparable ranges of $\alpha \mathrm{s}$, suggesting universal behaviour in the way Nature 'packs' the energy eigenvalues into 

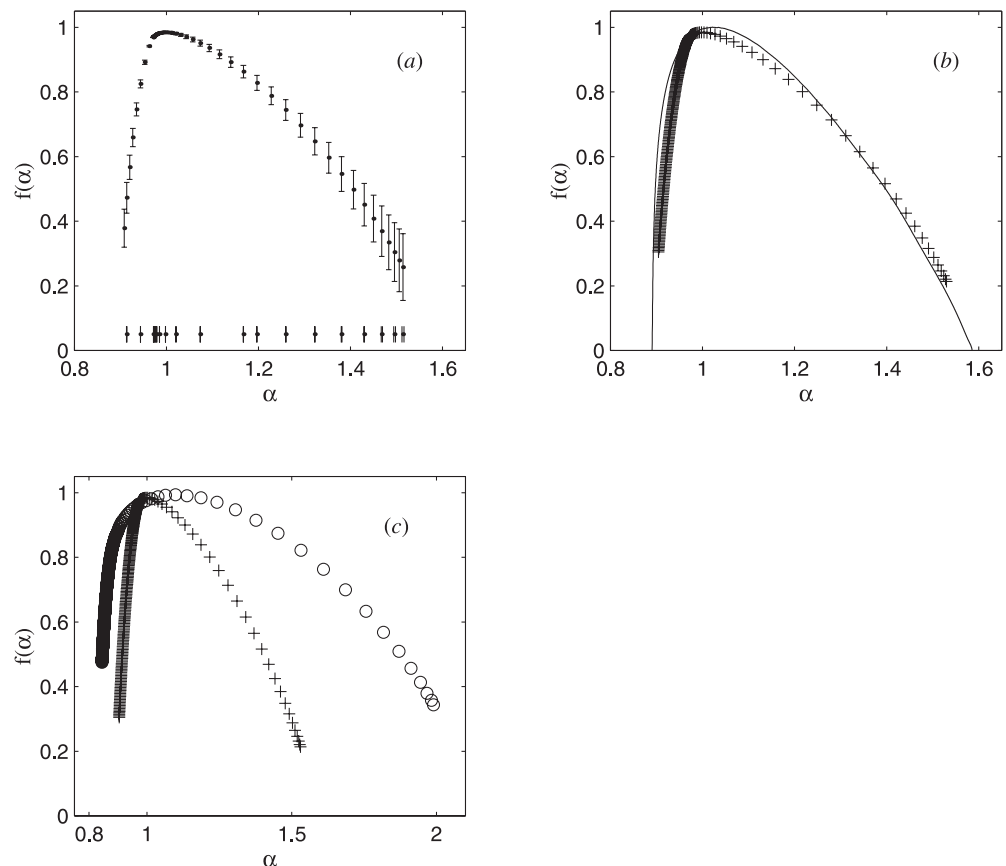

Figure 28. $f(\alpha)$ spectra of ( $a)$ unfolded Ce I; $(b)$ 'experimental' (+) and 'numerical' (full) unfolded $\mathrm{Ce}$ I; $(c)$ pre-unfolded $\mathrm{Ce}_{\mathrm{I}}$ (circles) and unfolded $\mathrm{Ce}_{\mathrm{I}}(+)$.

a given energy region. This 'packing' implies an efficiency law or minimization principle to which Nature subscribes. However, multifractality is suggestive of a quite complex set of interwoven scaling subsets. Why this complexity should be 'efficient' is another question. Of course, this may be related to maximizing the entropy (entropy = information loss/lack of information content) of the system, which in turn minimizes the information content of the system.

The $f(\alpha)$ spectra were then simulated using a binomial multiplicative process with a continuous support for the measure. It was found that the underlying scaling structure of the $f(\alpha)$ spectra could be understood in terms of a simple recursive Cantor set with two length scales and two probability scales. The numerical and experimental $f(\alpha)$ spectra were found to coincide for $\alpha$ s corresponding to the denser regions.

The effect of unfolding energy levels before calculating an $f(\alpha)$ spectrum was tested on Ce I. Since the $\alpha$ range of the $f(\alpha)$ spectrum was found to be more restricted in extent than that found for the pre-unfolded levels, this would imply that the unfolding (rescaling) process is actually a smoothing procedure. Thus, any extreme fluctuations in the unfolded values are reduced quite significantly.

Other lanthanide elements such as La, Pr, Eu and Dy were also tested for multifractality, but these did not yield any definitive results. Whether or not multi-scaling behaviour occurs across the whole of the periodic table has yet to be determined.

\section{Acknowledgment}

This work was supported by the Irish Science and Technology agency Enterprise Ireland under research grant no SC-99-206. 

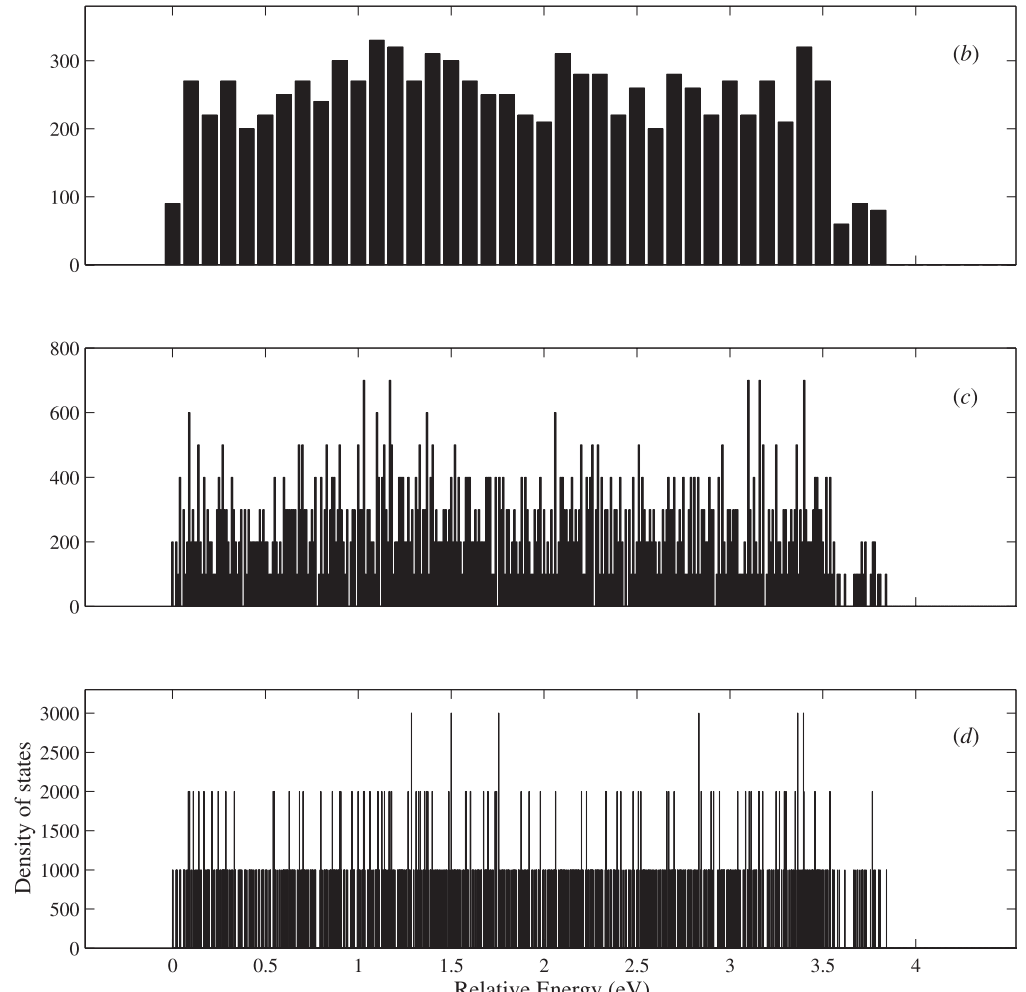

Figure 29. The density of states for unfolded $\mathrm{Ce}$ I: (a) eigenvalue spectrum; (b) $\Delta E=0.1 \mathrm{eV}$; (c) $\Delta E=0.01 \mathrm{eV} ;(d) \Delta E=0.001 \mathrm{eV}$.

\section{References}

[1] Mandelbrot B B 1977 Fractals: Form, Chance and Dimension (San Francisco, CA: Freeman)

[2] Mandelbrot B B 1982 The Fractal Geometry of Nature (New York: Freeman)

[3] Jensen M H, Kadanoff L P, Libchaber A, Procaccia I and Stavans J 1985 Phys. Rev. Lett. 55 2798-801

[4] Mandelbrot B B, Evertsz C J G and Hayakawa Y 1990 Phys. Rev. A 424528

[5] Hanan W, Heffernan D M and Earnshaw J 1998 Chaos Solitons Fractals 9875

[6] Einstein A J, Wu H and Gil J 1998 Phys. Rev. Lett. 80 397-400

[7] Ivanov P C, Amaral L A N, Goldberger A L, Rosenblum M G, Struzik Z R and Stanley H E 1999 Nature 399 $461-5$

[8] Jin Y, Sonagtao L and Guozhen W 1999 Chem. Phys. Lett. 301 217-22

[9] Sáiz A and Martínez V J 1996 Phys. Rev. E 54 2431-7

[10] Sáiz A and Martínez V J 1999 Phys. Rev. E 604993

[11] Carpena P, Coronado A V and Bernaola-Galvan P 2000 Physica A 287 37-48

[12] Halsey T C, Jensen M H, Kadanoff L P, Procaccia I and Shraiman B I 1986 Phys. Rev. A 33 1141-51 
[13] Pawelzik K and Schuster H G 1987 Phys. Rev. A 351

[14] Flambaum V V, Gribakina A A, Gribakin G F and Kozlov M G 1994 Phys. Rev. A 50 267-96

[15] Flambaum V V, Gribakina A A and Gribakin G F 1998 Phys. Rev. A 58 230-7

[16] http://physics.nist.gov/cgi-bin/AtData/main_asd

[17] Hamburger D, Biham O and Avnir D 1996 Phys. Rev. E 53 3342-58

[18] Feigenbaum M J, Jensen M H and Procaccia I 1986 Phys. Rev. Lett. 57 1503-6

[19] Chhabra A B, Jensen R V and Sreenivasan K R 1989 Phys. Rev. A $404593-611$

[20] Cowan R D 1981 The Theory of Atomic Structure and Spectra (Berkeley, CA: University of California Press)

[21] Friedrich H 1991 Theoretical Atomic Physics (Berlin: Springer)

[22] Mandelbrot B B 1979 C. R. Acad. Sci., Paris A 28881 